\title{
Qualitätsmessung mit Routinedaten in deutschen Pflegeheimen: Eine erste Standortbestimmung
}

\author{
Antje Schwinger, Susann Behrendt, Chrysanthi Tsiasioti, Kai Stieglitz, \\ Thorben Breitkreuz, Thomas Grobe und Jürgen Klauber \\ (c) Der/die Autor(en) 2018 \\ K. Jacobs et al. (Hrsg.), Pflege-Report 2018 \\ https://doi.org/10.1007/978-3-662-56822-4_10
}

\section{Zusammenfassung}

In Kontrast zu 780.000 hochbetagten und multimorbiden Menschen in deutschen Pflegeheimen steht die umfassend untersuchte Fehlversorgung in diesen Einrichtungen. Der Optimierungsbedarf zur Versorgungstransparenz und-qualität ist erheblich. Ausgehend von der These, dass ein multidimensionaler, sektoren-und berufsübergreifender Blick auf die Versorgungsqualität im Pflegeheim erforderlich ist, testeten die Autoren erstmals in Deutschland die Nutzung von Routinedaten der Kranken- und Pflegekassen (AOK) für diesen Kontext. Hierfür wurden insgesamt sechs Kennzahlen zur Arzneimittelversorgung, zu nosokomialen Erkrankungen, zu Hospitalisierungen sowie zur haus- und fachärztlichen Versorgung im Pflegeheim entwickelt und empirisch getestet. Im Ergebnis zeigt sich, dass die Operationalisierung routinedatenbasierter Qualitätskennzahlen für das stationäre Pflegesetting machbar ist. Auch die zum Teil erheblichen Versorgungsunterschiede zwischen den Pflegeheimen werden transparent gemacht. Auf der Agenda stehen nun die methodische Schärfung und Risikoadjustierung, damit langfristig routinedatenbasierte Qualitätsindikatoren die Entwicklung der pflegeheiminternen Qualität und die Versorgungstransparenz für die Pflegekassen empirisch unterstützen können.

In contrast to 780,000 elderly and multimorbid people in German nursing homes, there is an extensively analysed poor care in these facilities. The need for an improved transparency and quality of care is considerable. Based on the assumption that a multidimensional, cross-sectoral and cross-occupational view of the quality of care in nursing homes is called for, the authors tested the use of routine data from health and nursing insurance funds (AOK) for the first time in Germany for this context. For this purpose, a total of six key data on provision of medicines, nosocomial diseases, hospitalisations and general and specialist care in nursing homes were developed and empirically tested. The results show that the operationalisation of routine data-based quality indicators for inpatient care setting is feasible. The sometimes considerable differences in care between nursing homes are also made transparent. Methodological sharpening and risk adjustment are now on the agenda so that routine data-based quality indicators can empirically support the development of nursing home quality and provide transparency for the nursing care insurance funds in the long run. 


\subsection{Einführung}

Rund 780.000 Menschen leben heute dauerhaft in vollstationären Pflegeeinrichtungen und sind in der Regel hochbetagt, multimorbid und als besonders vulnerabel einzustufen (Statistisches Bundesamt 2017). Mehr als zwei Drittel der Pflegeheimbewohner besitzen neben somatisch bedingten Beeinträchtigungen eine eingeschränkte Alltagskompetenz, verbunden mit erheblichen kognitiven und kommunikativen Defiziten (Schwinger et al. 2017a). Sie sind stark abhängig von der Versorgung durch Dritte und erfahren durch die Aufgabe der eigenen Häuslichkeit und oftmals auch des sozialen Umfelds einen Verlust an Autonomie. Diese komplexen Bedarfslagen stehen einer umfassend untersuchten Fehlversorgung in deutschen Pflegeheimen gegenüber. Aufgezeigt werden u. a. eine unzureichende Wundversorgung, Schmerzerfassung und Dekubitusprophylaxe (MDS 2018), risikobehaftete Arzneiverordnungen, eine Fehlversorgung mit Psychopharmaka (Balzer et al. 2013; Schwinger et al. 2017a; Schwinger et al. 2016; Thürmann 2017), potenziell vermeidbare Krankenhauseinweisungen, Hospitalisierungen in der letzten Lebenswoche (Ramroth $\mathrm{H}$ 2006; Wiese et al. 2016) sowie eine defizitäre haus-, fach- und zahnärztliche Versorgung (Balzer et al. 2013; Kleina et al. 2017; Nitschke und Micheelis 2016; Rothgang et al. 2008; Rothgang et al. 2014; Schwinger et al. 2017a).

Das Potenzial für eine Verbesserung der Transparenz und Qualität des Versorgungsgeschehens in Pflegeheimen ist damit erheblich. Der Gesetzgeber hat als Reaktion die Vorgaben zur gesetzlichen Qualitätssicherung in der Langzeitpflege in den letzten zehn Jahren grundlegend weiterentwickelt (siehe auch Büscher et al, \ Kap. 4 im vorliegenden Band). Das Pflege-Neuausrichtungs-Gesetz (2012) stellte die Weichen für ein indikatorengestütztes Verfahren und richtete den Blick erstmals auf die Ergebnisqualität der Versorgung. Ausgangspunkt war ein vom Bundesministerium für Familie, Senioren, Frauen und Jugend (BMFSFJ) und vom Bundesgesundheitsministerium (BMG) beauftragtes Projekt, das ein Set von 15 pflegebezogenen Indikatoren zur Beurteilung der Ergebnisqualität in der stationären Langzeitpflege entwickelte (Wingenfeld et al. 2011). Die drei Pflegestärkungsgesetze (2015/2017) kon- kretisierten die Rahmenvorgaben sowie die wissenschaftliche Weiterentwicklung dieser nach dem damaligen Studienautor benannten »WingenfeldIndikatoren «. Es zeichnet sich derzeit ab, dass zehn bis zwölf Indikatoren mit engem Bezug zur pflegerischen Versorgung im Pflegeheim implementiert werden (Broge et al., \ Kap. 12 im vorliegenden Band). Datengrundlage der neuen Ergebnisindikatoren bilden durch die Pflegeheime gesondert $\mathrm{zu}$ dokumentierende Angaben zur Qualitätssicherung. Der Einbezug von Routinedaten der gesetzlichen Kranken- und Pflegeversicherung (GKV und SPV) ist nicht vorgesehen.

Im Lichte der oben skizzierten Versorgungsdefizite in der vollstationären Langzeitpflege wirft dies die Frage auf, ob das Potenzial an Versorgungsoptimierung damit ausreichend durch empirische Bestandsaufnahmen gestützt wird. Die gesetzlichen Weiterentwicklungen drehen sich maßgeblich um die Qualität von Versorgungsergebnissen, die im engeren Sinne den SGB XI-Leistungserbringern zuschreibbar sind. Die These des vorliegenden Beitrags aber lautet: Da die Versorgungsdefizite multikausal und damit sektoren- und vor allem auch berufsgruppenübergreifend zu verorten sind, bedarf es auch einer sektoren- und berufsgruppenübergreifenden Herangehensweise bei der Messung der Ergebnisqualität. Dies reiht sich ein in den Forschungsstand zur Mehrdimensionalität von Pflegequalität, der neben adäquater pflegerischer Performanz i. e. S. deren wechselseitige Relation mit einer Vielzahl von Einrichtungsmerkmalen wie z. B. der Heimstruktur, Führungskultur, der intra- und interpersonellen Zusammenarbeit und dem Qualifikationsmix des Personals benennt (Gallagher und Rowell 2003; Heslop 2014; Nakrem et al. 2009; Savitz et al. 2005).

Die hier erprobten ersten Annäherungen an routinedatenbasierte Qualitätskennzahlen für die stationäre Langzeitpflege sind im ersten Schritt für den Einsatz in der internen Qualitätsentwicklung im Pflegeheim sowie für eine verbesserte Versorgungstransparenz für die Pflegekassen gedacht. Der Einsatz der Kennzahlen soll dazu dienen, Defizite aufzudecken, Erfolge von Maßnahmen zu messen sowie einen berufsgruppenübergreifenden Qualitätsdiskurs anzustoßen und empirisch zu unterlegen. Die ggf. mögliche weitere Nutzung einiger 
der routinedatenbasierten Indikatoren für die externe Qualitätsberichterstattung ist erst $\mathrm{zu}$ einem späteren Zeitpunkt - bei hinreichender Reifung der methodischen Fundierung der Indikatoren - zu diskutieren.

Nach einer Kurzerläuterung der verwendeten Daten elaboriert der Beitrag insgesamt sechs Kennzahlen für relevante Versorgungsaspekte im Bereich der Arzneimittelversorgung, für nosokomiale Erkrankungen, Hospitalisierungen sowie die hausund fachärztliche Versorgung im Pflegeheim. International, insbesondere in Kanada und den USA, werden alle hier ausgewählten Versorgungsaspekte seit längerem als Indikatoren der externen Qualitätsberichterstattung in der Langzeitpflege herangezogen. ${ }^{1}$ Je ausgewählter Kennzahl geht der Beitrag auf deren Relevanz für die Qualitätssicherung in der Pflege ein, skizziert deren Operationalisierung und führt die Ergebnisse der Analysen zusammen. Den Abschluss des Beitrags bildet eine kennzahlenübergreifende Diskussion zur möglichen Weiterentwicklung derartiger Kennzahlen hin zu routinedatenbasierten Qualitätsindikatoren für die stationäre Langzeitpflege.

\subsection{Ausgewählte Versorgungs- aspekte der stationären Langzeitpflege für eine routine- datenbasierte Qualitätsmessung}

\subsubsection{Dekubitus im Pflegeheim}

Die Vermeidung und Behandlung von Druckgeschwüren (Dekubiti) sind zentrale Aufgaben der Pflege und Bestandteil der Qualitätsprüfungen des Medizinischen Dienstes der Krankenversicherung

\footnotetext{
1 Siehe z. B: Dekubitus - Kanada (HQO 2015), USA (CMS 2018); Harnwegsinfektion - USA (CMS 2018); Antipsychotika Kanada (HQO 2015), USA (CMS 2018), Niederlande (Gezondheidszorg 2013); ärztliche Versorgung - Schweden (Sveriges Kommuner och Landsting och Socialstyrelsen 2015); ambulant-sensitive Hospitalisationen - Kanada (HQO 2015). Anzumerken ist, dass die Indikatoren in keinem der hier genannten Länder über Routinedaten (claims data) operationalisiert sind, sondern auf Eigendokumentation der Heime, Befragungen und Informationen aus Prüfungen der Aufsicht beruhen. In diesem Sinne wird im Beitrag also Neuland betreten.
}

(MDK) nach $\$ 114$ SGB XI. Die in Form von PflegeQualitätsberichten veröffentlichten Ergebnisse zeigten im Jahr 2016 bei 3,9\% der Pflegeheimbewohner in Deutschland einen Dekubitus; bei knapp der Hälfte der Bewohner (43,7 \%) waren prophylaktische Maßnahmen erforderlich, von denen ein Viertel (19,3\%) diese Leistungen jedoch nicht erhielten (MDS 2018). In weiteren für den Beitrag rezipierten epidemiologischen Erhebungen variieren die Prävalenzen der (in der Einrichtung erworbenen) Dekubiti in deutschen Pflegeheimen zwischen rund $2 \%$ und $4 \%$ (DNQP 2017; Engelhart et al. 2009; Kottner et al. 2011; Wischnewski et al. 2011). ${ }^{2}$

Die Mehrzahl der Druckgeschwüre sind lagerungsbedingten Faktoren zuzuschreiben (Leffmann et al. 2005). Grunderkrankungen wie Diabetes mellitus und Inkontinenz, Beeinträchtigungen der Mobilität, der Durchblutung der Haut bzw. deren Schädigung gelten ebenso wie Stoffwechselstörungen, Dehydration (unzureichende Flüssigkeitszufuhr) und Malnutrition als wesentliche bewohnerspezifische Risikofaktoren (DNQP 2017; NPUAP et al. 2014). Dekubiti haben in Abhängigkeit vom Schweregrad erhebliche Folgen für die Betroffenen. Knochenabszesse und Sepsis durch infizierte Wunden, Schmerzen, ein unangenehmer Geruch sowie eine starke psychische Belastung können die Lebensqualität des Betroffenen erheblich mindern (DNQP 2015; Leffmann et al. 2005).

Gleichzeitig bestehen Standards für Prävention und Versorgung von Dekubitus im pflegerischen Setting, zu denen u. a. ein systematisches Risikoassessment ebenso wie Schulungen des Betroffenen und seiner Angehörigen, Bewegung sowie druckentlastende und -verteilende Maßnahmen zählen (DNQP 2015; DNQP 2017). Das deutsche Netzwerk für Qualitätsentwicklung in der Pflege geht im kürzlich aktualisierten Expertenstandard Dekubitusprophylaxe davon aus, »dass das Auftreten eines Deku-

2 Dieses Spektrum an Prävalenzangaben lässt sich zu einem großen Teil durch das variierende methodische Design der zugrunde liegenden Studien erklären (teils Ausschluss von leichten Dekubitusfällen, Einbezug ambulanter Einrichtungen, Messverfahren, Berechnung unterschiedlicher Prävalenzarten, Adjustierungen, regionale Analysen etc.). Vgl. hierzu auch Kottner et al., Literaturstudie, S. 50-93, in DNQP 2017. 
bitus weitgehend verhindert werden kann « (DNQP 2017, S. 19). Als unerwünschtes Ereignis in der Gesundheitsversorgung ist Dekubitus unter diesem Aspekt seit Jahren ein wichtiger Indikator im Kontext der Qualitätssicherung nach $\$ 114$ SGB XI ff. ${ }^{3}$. Auch im bereits angesprochenen Forschungsprojekt von Wingenfeld et al. (2011) mit dem Ziel der Entwicklung indikatorengestützter Messverfahren zur Ergebnisqualität in der stationären Altenhilfe bildet die Dekubitusentstehung in Abhängigkeit von der Fähigkeit zum Positionswechsel im Bett zwei von 15 der empfohlenen Qualitätsindikatoren (Wingenfeld et al. 2011).

\subsubsection{Harnwegsinfektionen im Pflegeheim}

Harnwegsinfektionen (HWI) gehören zu den häufigsten nosokomialen Infektionen im Pflegeheim. Im Rahmen der HALT-2-Studie (Healthcare-associated infections in long-term care facilities, 2. Erhebung) des European Centre for Disease Prevention and Control (ECDC 2013) ergaben Punktprävalenzmessungen von Infektionen in deutschen Pflegeheimen, dass es sich bei knapp einem Drittel $(28,4 \%)$ aller aufgetretenen Infektionen um HWI handelte $(0,8 \%$ der 17.208 Pflegeheimbewohner der Studie), in 90,1 \% lagen jedoch keine mikrobiologischen Befunde vor (Ruscher et al. 2015). Einigkeit besteht darin, dass betagte Pflegeheimbewohner, insbesondere Frauen, sowohl ein hohes Risiko für HWI aufweisen als auch ein hohes Risiko für Komplikationen, Folgeerkrankungen, rezidivierende Infektionen sowie die Ausbildung von Antibiotikaresistenzen (Becher et al. 2015; DGU 2017; KRINKO 2015; Wischnewski et al. 2011). Neben diesen intrapersonellen Risikofaktoren begünstigen unzureichende Präventivmaßnahmen, insbesondere zur Händehygiene und zum Umgang mit Blasenkathetern, das Entstehen von HWI. Die Kommission für Krankenhaushygiene und Infektionsprävention (KRINKO) aktualisierte erst 2016 ihre Empfehlungen zur Hängehygiene und bezog dabei

3 Auch im Kontext der externen Qualitätssicherung im Krankenhaus nach § 137 SGB V (G-BA 2018) findet die Messung von Dekubita Eingang. explizit auch Pflegeheime ein (KRINKO 2016). Auch wenn die Verantwortung für diese Infektionen nicht allein bei der pflegerischen Performanz liegt bzw. der Anteil der durch eine adäquate Pflege vermeidbaren Erkrankungsfälle nicht quantifizierbar erscheint (Wingenfeld et al. 2011), so können Harnwegsinfekte sehr wohl als "pflegeheimassoziierte Erkrankungen «bzw. »als ein Parameter der hygienischen Ergebnisqualität in Altenpflegeheimen" gesehen werden (Engelhart et al. 2009, S. 938, Ruscher et al. 2012).

Ein wichtiges Argument, einen entsprechenden Indikator zu bilden, lässt sich aus Surveillance-Systemen für nosokomiale Infektionen, in Deutschland gemäß $\$ 23$ Abs. 1 Infektionsschutzgesetz (IfSG), per se ableiten: Das Aufzeigen von in der Einrichtung erworbenen Infektionen führt langfristig zur Reduktion der Infektionsraten, selbst wenn die endemischen nosokomialen Infektionen, so KRINKO, nur partiell durch entsprechende Hygienemaßnahmen vermeidbar seien (KRINKO 2001).

\subsubsection{Versorgung mit Antipsychotika im Pflegeheim}

Im Jahr 2015 hatten rund drei von vier (73,0 \%) der stationär versorgten Pflegebedürftigen im Alter von mindestens 60 Jahren eine im Sinne der Pflegeversicherung anerkannte eingeschränkte Alltagskompetenz (EA) und somit i. d. R. eine Demenz (- Tab. 10.1). Damit einher gehen psychische und Verhaltenssymptome wie Angst, Aggressivität oder Verhaltensweisen wie "Schreien « und "Umherwandern«, die - mit variierender Häufigkeit und Dauer - bei jedem Betroffenen im Erkrankungsverlauf auftreten (Cerejeira et al. 2012; James 2011). Die mit diesen Symptomen assoziierte Verabreichung von Antipsychotika für ältere, dementiell erkrankte Menschen ist mit erheblichen Risiken $u$. a. für zerebrovaskuläre Ereignisse und einer erhöhten Mortalität insgesamt sowie Nebenwirkungen wie Gangstörungen, Stürzen und weiterer kognitiver Beeinträchtigung behaftet (DGPPN und DGN 2016; Kirkham et al. 2017). Der entsprechende Medikationsnutzen gilt demgegenüber als moderat. Vor diesem Hintergrund empfiehlt die S3-Leitlinie »Demenzen« der Deutschen Gesellschaft für Psy- 
chiatrie und Psychotherapie, Psychosomatik und Nervenheilkunde (DGPPN) sowie der Deutschen Gesellschaft für Neurologie (DGN) nur einen kurzen, befristeten Einsatz spezifischer atypischer Antipsychotika in geringstmöglicher Dosis. Eine engmaschige Kontrolle des Behandlungsverlaufs ist dabei essentiell (DGPPN und DGN 2016). Als erstes Mittel der Wahl empfiehlt die Leitlinie nicht-medikamentöse Ansätze (u. a. Validation, kognitive Verfahren, sensorische Stimulation, Beschäftigungsangebote, Bewegungsförderung) (Bartholomeyczik et al. 2006; DGPPN und DGN 2016; James 2011). Die Wirksamkeit einiger Verfahren wie der verstehenden Diagnostik, der Schulung des Personals und der Angehörigen wie auch weiterer verhaltensbezogener Ansätze gilt als belegt (DGPPN und DGN 2016; Dickson et al. 2012). Ferner zeigen internationale und nationale Studien, dass Interventionen zur besseren Zusammenarbeit der versorgungsrelevanten Berufsgruppen Verordnungsraten von Antipsychotika bei herausforderndem, dementiell bedingtem Verhalten reduzieren können (Bartholomeyczik et al. 2010; Coon et al. 2014; Kuhlmey et al. 2010; Thürmann et al. 2010). Trotzdem schätzen Studien zum Einsatz von Antipsychotika in Pflegeheimen je nach Betrachtung der Bewohner ohne oder mit Demenz - die Verordnungsraten auf $28 \%$ bis $47 \%$ (Bergner 2016; Mauleon et al. 2014; Molter-Bock et al. 2006; Richter et al. 2012). Aus einer Meta-Analyse von 43 internationalen Untersuchungen zur stationären Pflege sind Raten von $24 \%$ bis $64 \%$ bezogen auf dementiell erkrankte Pflegeheimbewohner bekannt (Kirkham et al. 2017).

Dies unterstreicht die hohe Relevanz des Ausmaßes des Antipsychotika-Einsatzes für die Qualitätssicherung in der stationären Langzeitpflege: Das Qualitätsziel ist grundsätzlich beeinflussbar. Gestützt wird dies erstens durch die gute Evidenz, dass das Absetzen der Antipsychotika bei den meisten Betroffenen keine wesentliche Symptomverschlechterung bedingt (Declercq et al. 2013). Es sind zweitens die Pflegekräfte, die im alltäglichen Umgang mit den teilweise herausfordernden Symptomen regelmäßig ärztliche Verordnungen initiieren (Schwinger et al. 2017b). Drittens sind eine Vielzahl der nicht-medikamentösen Ansätze pflegebezogen (z. B. verstehende Pflegeansätze, Validation) bzw. durch organisatorische Abläufe (z.B. Fallbe- sprechungen, Austausch mit Ärzten) oder Strukturen (Betreuungs- und weitere Therapieangebote) auf Pflegeheimebene steuerbar.

\subsection{4 Ärztliche Versorgung im Pflegeheim}

Pflegebedürftige sind angesichts ihrer somatischen und psychischen (Multi-)Morbidität und gesundheitlichen Bedarfslagen in der Regel auf Unterstützung bei der Inanspruchnahme von ambulantärztlichen Leistungen angewiesen und oft nicht in der Lage, ihre Beschwerden adäquat zu kommunizieren (vgl. $>$ Abschn. 10.2.3). Mobilitäts- und kognitive Einschränkungen erfordern nicht selten eine Arztvisite im Pflegeheim. Die Bedeutung dieser Hemmnisse bei Arztkontakten wird durch zahlreiche Hinweise auf defizitäre ambulante haus- und fachärztliche Versorgung in der stationären Langzeitpflege unterstrichen. Problematisch sind insbesondere Polymedikation, risikobehaftete Verordnungen von für Ältere potenziell ungeeigneten Arzneimittel (sogenannte PRISCUS-Arzneiverordnungen) (Schwinger et al. 2017a; Schwinger et al. 2016) sowie eine Fehlversorgung mit Psychopharmaka und Antidementiva bei dementiellen Bewohnern (Balzer et al. 2013; Thürmann 2017). Aufgezeigte Defizite im Bereich Diagnostik und/oder Versorgung beziehen sich neben dementiellen Erkrankungen (Balzer et al. 2013) auf Depressionen (Gutzmann et al. 2017; Thürmann 2017) sowie Diabetes mellitus (Balzer et al. 2013; Schwarzkopf et al. 2017). Weitere »unmet needs" werden für die Bereich der Heilmittelversorgung und Mundgesundheit benannt (Kleina et al. 2017; Nitschke und Micheelis 2016; Rothgang et al. 2008; Rothgang et al. 2014). Mit Blick auf die Versorgungsdefizite wird immer wieder auf die hierfür ursächliche defizitäre Schnittstelle »ärztliche Versorgung im Pflegeheim«, d. h. die fehlende ärztliche Präsenz und die schlechte intra- und interprofessionelle Kommunikation hingewiesen (Balzer et al. 2013; Ruscher et al. 2012; SVR Gesundheit 2009; Wischnewski et al. 2011).

Der Gesetzgeber hat mit vielfältigen Anpassungen der Rahmenbedingungen das Problem zu adressieren versucht: Bereits 2008 wurden Kooperationsverträge zwischen Pflegeheimen und niedergelas- 
senen (Zahn-)Ärzten ermöglicht (\$ 119b SGB V), 2012 dann Zuschläge für Leistungen im Rahmen solcher Verträge eingeführt ( $\$ 87$ Abs. 2j SGB V), 2015 wurde die entsprechende »Kann«-Regelung zum Vertragsabschluss dann in eine "Muss «-Vorgabe umgewandelt. Die heutigen Rahmenvorgaben zur »kooperativen und koordinierten ärztlichen und pflegerischen Versorgung in stationären Pflegeheimen ${ }^{4}$ zielen $u$. a. auf die Optimierung der ArztPersonal-Kommunikation durch feste Ansprechpartner für Pflegeeinrichtungen $(\$ 119 \mathrm{~b}$ Abs. 2 SGB V). Dabei sind die Hausärzte für die »Durchführung und Koordination der medizinischen Versorgung der Versicherten in Abstimmung mit der stationären Pflegeeinrichtung und den ggf. beteiligten Fachärzten « verantwortlich ( $\$ 2$ Abs. 2 Satz 1 der Vereinbarung). Die Schnittstelle Arzt-Pflegeheim wird auch bei den derzeitigen MDK-Qualitätsprüfungen nach $\$ 114$ a SGB XI berücksichtigt - jedoch allein fokussiert auf die Kooperation zwischen Arzt und Pflegepersonal bei Bewohnern mit chronischen Schmerzen (GKV-SV 2017).

\subsubsection{Ambulant-sensitive Hospitalisierungen von Pflegeheimbewohnern}

Unter ambulant-sensitiven Hospitalisierungen werden jene Krankenhauseinweisungen gefasst, die so die zugrunde liegende These - durch »Vorsorge oder rechtzeitige Intervention im ambulanten Sektor" (Sundmacher und Schüttig 2015) nicht erforderlich wären. Nach US-amerikanischem Vorbild existiert seit einigen Jahren ein spezifischer deutscher Katalog ambulant-sensitive Behandlungsanlässe im Krankenhaus (ASK), basierend auf einer Kernindikationsgruppe (22 Krankheitsgruppen) und einer Gesamtindikationsliste (40 Krankheitsgruppen) (Sundmacher und Schüttig 2015; Weissman et al. 1992).

Die Ursachen für hohe ASK-Raten im Allgemeinen sind divers: Während der Zusammenhang

4 Anlage 27 zum Bundesmantelvertrag :»Vereinbarung nach $\S 119 \mathrm{~b}$ Abs. 2 SGB V zur Förderung der kooperativen und koordinierten ärztlichen und pflegerischen Versorgung in stationären Pflegeheimen«. von strukturellen Hürden im ambulanten Sektor und ASK mittlerweile als belegt gilt, steht die Klärung, inwieweit ASK-Raten tatsächlich ein Ausdruck von mangelnder ambulant-ärztlicher Behandlungsqualität sind, noch aus (Burgdorf und Sundmacher 2014; Rosano et al. 2013). Ferner kann der Patient selbst entscheidend dazu beitragen, das Motto ambulant vor stationär umzukehren. $\mathrm{Zu}$ den relevanten patientenbezogenen Faktoren zählen der sozioökonomische Status, die Morbidität, das Gesundheitsverhalten sowie individuelle Versorgungspräferenzen (Sundmacher und Schüttig 2015). Auch wenn zahlreiche nationale Studien bzw. Gutachten dieses Konzept seither aufgegriffen haben (Albrecht und Sander 2015; Fischbach 2016; Nagel et al. 2017), wurde eine spezifische Betrachtung der ASK-Fälle ausschließlich für Pflegebedürftige in deutschen Pflegeheimen bisher nicht adressiert. Demgegenüber geben mehrere internationale Studien Hinweise, dass potenziell vermeidbare Hospitalisierungen, zu denen ASK neben anderen zählen, in der stationären Langzeitpflege durchaus eine häufige Problematik und ein erhebliches Risiko für die Pflegebedürftigen darstellen (Burke et al. 2015; Kirsebom et al. 2015; Temple et al. 2017). Aufgrund der Risikostruktur der Pflegeheimbewohner können die Risiken eines Krankenhausaufenthalts erheblich sein. Neben somatischen Pathologien wie nosokomialen Infektionen (vgl. hierzu auch - Abschn. 10.2.2), Nebenwirkungen von Arzneimittelswitches oder auch durch Bettlägerigkeit provozierte Beschwerden führt die Hospitalisierung, d. h. die Unterbringung in heimfernen Räumlichkeiten mit unbekanntem Personal und ungewohnten Abläufen, und auch die Rückkehr ins Pflegeheim nicht nur bei dementiellen Bewohnern zu sogenannten »transfer traumas" bzw. "relocation stress" - verbunden mit Orientierungslosigkeit, Depression und damit einer ggf. stark beeinträchtigten Lebensqualität (Keville 1993; Kirsebom et al. 2015). Daraus abgeleitet testet der vorliegende Beitrag auch die Verwendung der heimbezogenen Häufigkeit von ASK als Kennzahl in der routinedatenbasierten Qualitätsmessung im Pflegeheimsetting. 


\subsection{Operationalisierung der Versorgungsaspekte auf Basis von Routinedaten der GKV und SPV}

\subsubsection{Datengrundlage}

Für die Analysen wurden anonymisierte versichertenbezogene Abrechnungsdaten des Jahres 2015 der 11 regionalen AOK Pflege- und Krankenkassen ausgewertet. Enthalten sind Informationen über Alter und Geschlecht, das Vorliegen einer Pflegestufe und ggf. zusätzlich einer eingeschränkten Alltagskompetenz (wie diese vor Umsetzung des neuen Pflegebedürftigkeitsbegriffs im Jahr 2017 definiert war), ferner Informationen zur ambulant-ärztlichen, stationären, Arzneimittel- und Hilfsmittelversorgung. Die Analysen erfolgten quartalsbezogen. Für das erste Quartal wurden AOK-Versicherte ( $\geq 60$ Jahre) erfasst, die bereits zu Quartalsbeginn (Januar) Leistungen der vollstationären Dauerpflege ( $\$ 43$ SGB XI) ${ }^{5}$ beansprucht haben. Analog dazu bildeten die Monate April, Juli und Oktober jeweils den Referenzzeitraum für den Einschluss der AOK-Versicherten in den Quartalen II bis IV (2015). Dies stellt mit Blick auf die jeweils quartalsbezogene Grundgesamtheit sicher, dass keine Versicherten eingehen, die während des Quartals (z. B. in den ersten beiden Monaten) u. a. auch ambulant gepflegt wurden oder nicht pflegebedürftig waren. Im Quartalsverlauf verstorbene Pflegeheimbewohner sind hingegen eingeschlossen.

Um die eindeutige Zuordnung des Versicherten zu einem Pflegeheim zu gewährleisten, wurden Bewohner, die im Lauf des Jahres das Pflegeheim wechselten, aus der Datengrundlage entfernt. Weitere Bereinigungen betrafen den Ausschluss von Pflegeheimen ohne eindeutiges Institutskennzeichen und ohne Pflegetransparenzbericht der vollstationären Prüfung. Um eine ausreichende Anzahl an Fällen pro Pflegeheim für die empirischen Analysen sicherzustellen, wurden ausschließlich Pflegeheime mit mindestens 20 AOK-versicherten Pflegeheimbewohnern in jedem Quartal einbezogen. Die

5 Personen in vollstationären Einrichtungen der Hilfe für behinderte Menschen (§ 43a SGB XI)
- Tab. 10.1 Deskriptive Übersicht der Pflegeheimbewohner ab 60 Jahre sowie der Pflegeheime nach Träger und Größe (2015) (Anteile in \%)

\begin{tabular}{|c|c|c|}
\hline & $\begin{array}{l}\text { Stich- } \\
\text { probe }\end{array}$ & $\begin{array}{l}\text { Pflege- } \\
\text { statistik }\end{array}$ \\
\hline \multicolumn{3}{|l|}{ Im Durchschnitt der Quartale } \\
\hline Frauen & 74,8 & 73,3 \\
\hline Männer & 25,2 & 26,7 \\
\hline \multicolumn{3}{|l|}{ Alter } \\
\hline $60-70$ & 6,4 & 6,9 \\
\hline $70-80$ & 18,4 & 20,5 \\
\hline 80 Jahre und älter & 75,3 & 72,6 \\
\hline \multicolumn{3}{|l|}{ Pflegestufe } \\
\hline Pflegestufe 0 & 0,8 & 2,0 \\
\hline mit Pflegestufe I & 37,1 & 38,1 \\
\hline mit Pflegestufe II & 41,3 & 39,8 \\
\hline mit Pflegestufe III + Härtefälle & 20,8 & 20,1 \\
\hline $\begin{array}{l}\text { Eingeschränkte Alltagskom- } \\
\text { petenz (PEA) im Sinne des } \\
\S 45 \text { a SGB XI (vor PSG II) }\end{array}$ & 69,2 & 73,0 \\
\hline \multicolumn{3}{|l|}{ Jahreskennzahl } \\
\hline $\begin{array}{l}\text { Anteil verstorbener Pflegeheim- } \\
\text { bewohner }\end{array}$ & 25,4 & - \\
\hline \multicolumn{3}{|l|}{$\begin{array}{l}\text { Verweildauer im Pflegeheim, } \\
\text { in Monaten }\end{array}$} \\
\hline Mittlere Verweildauer & 9,6 & - \\
\hline 25. Perzentil & 8,9 & - \\
\hline 75. Perzentil & 9,8 & - \\
\hline
\end{tabular}

Im 1. Quartal 2015

Träger

\begin{tabular}{|l|l|l|}
\hline öffentlich & 5,0 & 4,8 \\
\hline $\begin{array}{l}\text { freigemeinnützig } \\
\text { privat }\end{array}$ & 60,7 & 53,0 \\
\hline Größe & 33,9 & 42,2 \\
\hline 21-50 Plätze & 10,3 & 27,9 \\
\hline $51-150$ Plätze & 82,8 & 66,1 \\
\hline 151 und mehr Plätze & 6,9 & 6,0 \\
\hline
\end{tabular}

Quelle: AOK-Daten 2015, Pflegestatistik 2015 (Statistisches Bundesamt 2017) 
finale Stichprobe enthielt 5.622 vollstationäre Pflegeheime und 232.451 AOK-Versicherte. Damit gehen die Hälfte $(50,4 \%)$ aller deutschen Pflegeheime und fast jeder Dritte $(31,4 \%)$ bundesweit stationär Pflegebedürftige ab 60 Jahre in die Analysen ein. - Tab. 10.1 gibt eine Übersicht über Charakteristika der Untersuchungspopulation sowie der Pflegeheime im Vergleich zur Pflegestatistik des Statistischen Bundesamtes.

\subsubsection{Dekubitus im Pflegeheim}

Die Operationalisierung der Kennzahl »Dekubitusfälle pro Pflegeheimbewohner« erfolgte mehrstufig. Explorative Auszählungen gaben einen Überblick über die Häufigkeit von Bewohnern mit einer Dekubitus-Diagnose und mit einer Verordnung geeigneter Verbandsmaterialien zur Wundversorgung bzw. von dekubitusspezifischen Hilfsmitteln im Durchschnitt der Quartale im Referenzjahr 2015. Inhaltlich ausschlaggebend für das Einbeziehen von Verbandsmaterial zur Wundversorgung sowie von Hilfsmitteln waren die Ausführungen in den Expertenstandards zur Dekubitusprophylaxe und zur Versorgung von chronischen Wunden sowie in den einschlägigen Leitlinien (DNQP 2015; DNQP 2017; NPUAP et al. 2014). Bei einem vorliegenden Dekubitus ab Grad II ist unbedingt und unverzüglich eine Wundversorgung mit geeignetem Verbandsmaterial durchzuführen und bei Bedarf durch druckverteilende Hilfsmittel zu ergänzen. Die jeweils zugrundeliegenden Definitionen und Klassifikationen zeigt - Tab. 10.2. Zur Identifizierung der Dekubitusfälle als im Pflegeheim erworbene Erkrankungen werden ausschließlich die ambu- lant-ärztlichen Diagnosen mit Zusatzkennzeichen G (gesicherte Diagnose) betrachtet.

- Abb. 10.1 fasst die Ergebnisse der Operationalisierung zur Kennzahl »Dekubitusfälle je Bewohner im Jahr« zusammen. Der Anteil Pflegeheimbewohner mit Dekubitusdiagnose beträgt im Durchschnitt der Quartale 9,2 \% und damit deutlich mehr als aus epidemiologischen Studien bekannt. Lediglich $4 \%$ der Pflegeheimbewohner haben sowohl eine Verordnung eines Verbandsmaterials und eine Diagnose; eine dekubitusspezifische Hilfsmittelverordnung und Diagnose wiesen im Referenzjahr sogar nur 1,4\% auf. Anders formuliert findet sich bei $84,7 \%$ der Bewohner mit Dekubitus-Diagnose keine (präventive und/oder behandlungsrelevante) Verordnung von dekubitusspezifischen Hilfsmitteln. Da davon auszugehen ist, dass bestimmte Hilfsmittel wie Weichlagerungskissen im Pflegeheim bereits verfügbar sind, bei Bedarf ausgeteilt und damit nicht in den Hilfsmittelverordnungsdaten erscheinen, andere wiederum präventiv verordnet werden (DNQP 2017), wird auf den Einbezug dieser Information zur Diagnosevalidierung über Routinedaten vorerst verzichtet. Für mehr als die Hälfte (56,6 \%) der Bewohner mit Diagnose ist zudem keine Verordnung für Verbandsmaterial dokumentiert. Es ist insofern wahrscheinlich, dass es sich bei einem großen, jedoch nicht quantifizierbaren Anteil an Dekubitusdiagnosen um »inaktive" Diagnosen oder Fehlkodierungen handelt. Eine Operationalisierung des Dekubitus allein auf Basis der vertragsärztlichen Diagnosen erscheint insofern nicht angebracht.

Entschieden wurde sich vor diesem Hintergrund für eine Operationalisierung auf Basis von Diagnosen und Verordnungen von Verbandsmate-

- Tab. 10.2 Dekubitus - relevante Klassifikationen zur Identifizierung auf Routinedatenbasis

\begin{tabular}{|l|l|l|l|}
\hline & Diagnose Dekubitus & Verbandsstoffe & Hilfsmittel \\
\hline Klassifikation & ICD-10-GM & Pharmazentralnummer (PZN) & $\begin{array}{l}\text { Hilfsmittelverzeichnis der GKV } \\
\text { (HMV) }\end{array}$ \\
\hline $\begin{array}{l}\text { Definition } \\
\text { Daten }\end{array}$ & $\begin{array}{l}\text { L89*-Dekubitalgeschwür und } \\
\text { Druckzone }\end{array}$ & $\begin{array}{l}\text { Hydroaktive Wundauflagen, } \\
\text { vgl. Tab. 10.9 (Anhang) }\end{array}$ & $\begin{array}{l}\text { Hilfsmittel gegen Dekubitus, } \\
\text { Gruppe 11 }\end{array}$ \\
\hline \begin{tabular}{l} 
Quelle: WIdO \\
\hline
\end{tabular} & Ambulante gesicherte Diagnosen & Ambulante Verordnungen & Ambulante Verordnungen \\
\hline
\end{tabular}



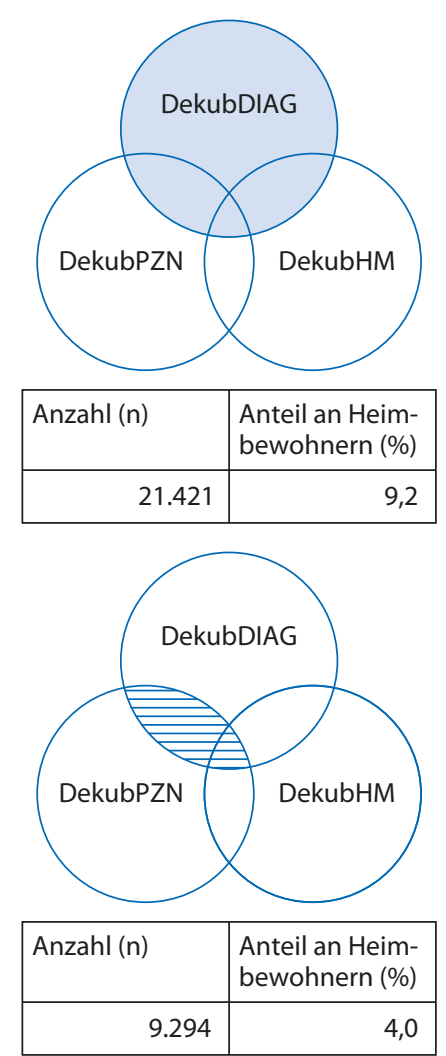
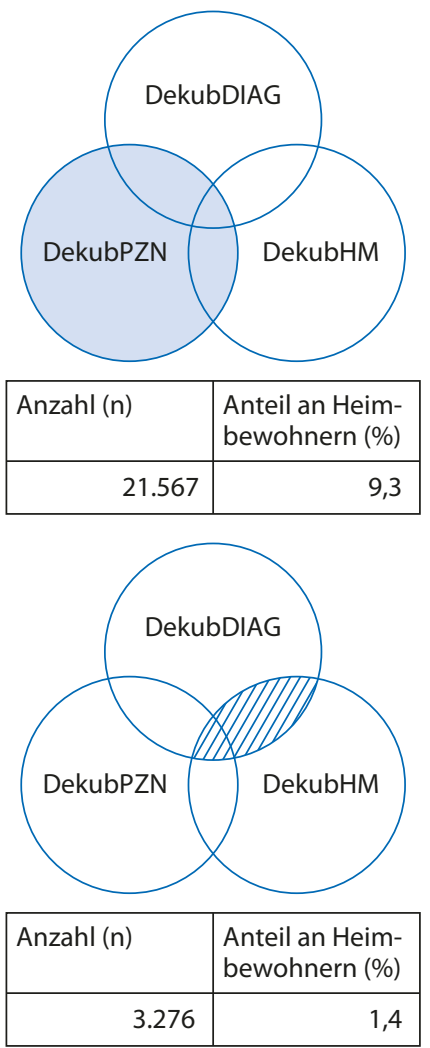
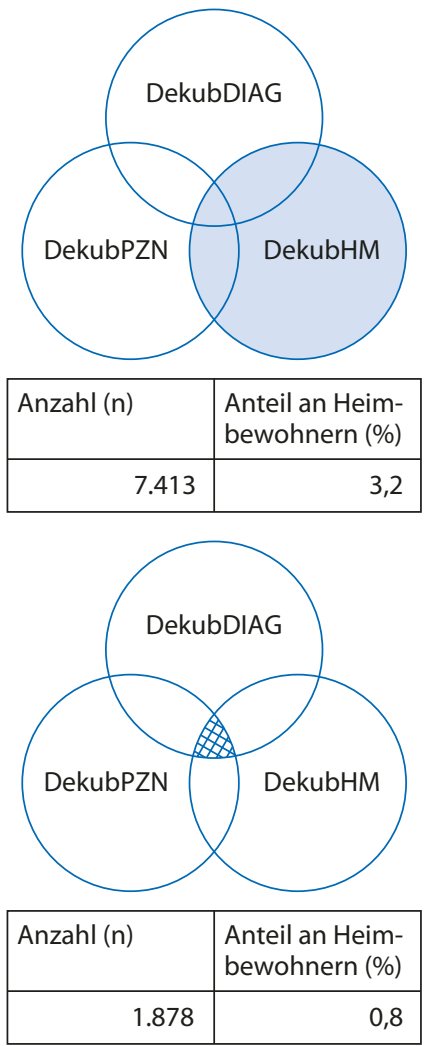

Pflege-Report 2018

Abb. 10.1 Häufigkeit von Dekubitus-Diagnosen (DIAG), Verordnungen von entsprechendem Verbandsmaterial (PZN) sowie von dekubitusspezifischem Hilfsmittel (HM) im Pflegeheim (2015, im Durchschnitt der Quartale) (Quelle: AOK-Daten 2015)

rial. - Tab. 10.6 listet die Merkmale der finalen Kennzahl auf. Zur Berechnung dieser Kennzahl wurde ferner auf ausschließlich neu aufgetretene Dekubitusfälle eingeschränkt. Solche liegen vor, wenn ein Bewohner in einem Quartal des Referenzjahres mindestens eine gesicherte ambulante Diagnose sowie mindestens eine Verordnung von Verbandsmaterial zur Wundversorgung aufweist. $\mathrm{Zu}-$ sätzlich darf der Bewohner im Vorquartal keine dieser ambulanten Diagnosen aufweisen (Inzidenzkriterium). Dieser Algorithmus limitiert die maximale Anzahl möglicher Dekubitusfälle pro Einwohner und Jahr auf zwei Ereignisse.

Die Referenzpopulation, d. h. die Versicherten, auf die sich die Dekubitusfälle beziehen, bilden alle Bewohner des Pflegeheims - unabhängig von ihren individuellen Risikofaktoren für Dekubiti. Unterschiede in den bewohnerindividuellen Verweildauern sind in der Stichprobe nicht groß, jedoch zu berücksichtigen: Bei dem Viertel der Pflegeheime mit den kürzesten Verweildauern (25\%-Perzentil) verblieben die Bewohner rund neun Monate oder kürzer, bei dem Viertel der Pflegeheime mit den längsten Verweildauern (75\%-Perzentil) mindestens zehn Monate (•Tab. 10.1). Ohne eine Berücksichtigung dieser Zeiten wären Pflegeheime mit kurzen Verweildauern durch die Berechnung einer Kennzahl unerwünschte Ereignisse in Relation zur Anzahl der Bewohner rein rechnerisch im Vorteil: Bei ihnen gingen im Nenner mehr Bewohner ein als bei solchen mit längeren Verweildauern (weniger Bewohner je Pflegeheimplatz). Um entsprechende 
Unterschiede zwischen den Einrichtungen auszugleichen, wurde je Pflegeheim die aufsummierte Gesamtverweildauer aller Bewohner in sogenannte Vollzeitäquivalente (Vollzeit-Verweildauer entspricht einem Jahr) umgerechnet. Die Kennzahl soll damit abbilden, wie viele unerwünschte Ereignisse (hier Dekubitus-Fälle) im Jahr je durchgängig belegten Pflegeheimplatz auftreten. Die im Folgenden verwendete Terminologie bei der Bezeichnung der Kennzahl lautet vereinfachend je Bewohner, meint jedoch den durchgängig belegten Pflegeheimplatz.

\subsubsection{Harnwegsinfektionen im Pflegeheim}

Analog zur Kennzahl »Dekubitus-Fälle je Bewohner« prüften explorative Auszählungen als ersten Schritt der Operationalisierung die Validität der Diagnose Harnwegsinfekt (HWI) in den Routinedaten. Dabei wurden neben den Diagnosedaten zusätzlich Verordnungen der für den Harnwegsinfekt indizierten Antibiotika einbezogen (• Tab. 10.3). Während asymptomatische Bakterien mit wenigen Ausnahmen nicht therapiert werden, zielt die Behandlung von symptomatischen Harnwegsinfektionen auf die Beseitigung der infektionsverursachenden Bakterien, auf die symptomatische Therapie sowie auf die Verhinderung einer Beeinträchtigung des Organsystems (Fünfstück 2011; DGU 2017). Die hier gewählte Validierung der Diagnosen setzt jedoch auf der Hypothese auf, dass bei betagten Menschen in Pflegeeinrichtungen angesichts von Alter, Morbidität und reduzierter Immunabwehr regelhaft davon Abstand genommen wird, eine Spontanheilung abzuwarten und damit auf Medikation zu verzichten (vgl. auch DGU 2017). Durch die Validierung der Diagnosen sollen falsch-positive Fälle ausgeschlossen und die Spezifität der Messung erhöht werden. Eine behandelte (und damit symptomatische) Harnwegsinfektion, so die Annahme, ist mit hoher Wahrscheinlichkeit eine tatsächlich vorliegende Infektion. Zur spezifischen Betrachtung der im Pflegeheim aufgetretenen Harnwegsinfektionen sind die Haupt- und Nebendiagnosen aus Krankenhausaufenthalten der Bewohner kein Bestandteil der Analyse.

Im Durchschnitt der Quartale wiesen rund 7,5\% der Bewohner im Referenzjahr 2015 eine Harnwegsinfektion als ambulant-ärztliche gesicherte Diagnose auf; davon wurden $62,0 \%$ (4,7 \% aller Bewohner) antibiotisch therapiert (• Abb. 10.2). Umgekehrt wiesen 15,2 \% eine Antibiotika-Verordnung auf, 30,7 \% von ihnen zudem eine Harnwegsinfekt-Diagnose. In der HALT-2-Studie fielen die HWI-Prävalenz mit $<1 \%$ und die Antibiotikagabe mit 1,9\% bedeutend geringer aus. Knapp die Hälfte $(44,9 \%)$ der verordneten Antibiotika bezogen sich dort auf die Therapie von Harnwegsinfektionen (Ruscher et al. 2015). Der Vergleich ist jedoch nur bedingt möglich. Die HALT-2-Studie beinhaltete eine Primärerhebung in Kooperation mit rund 220 freiwillig teilnehmenden Pflegeeinrichtungen. Die HWI-Diagnostik erfolgte symptombasiert analog zu den McGeer-Surveillance-Kriterien, der Bezug der verabreichten Antibiotika auf die Infektion war hier direkt erfragbar. Demgegenüber ist im Rahmen der vorliegenden Sekundärdaten-

\begin{tabular}{|c|c|c|}
\hline & Diagnose HWI & Antibiotika \\
\hline Klassifikation & ICD-10-GM & ATC \\
\hline Definition & $\begin{array}{l}\text { N10 Akute tubulointerstittielle Nephritis } \\
\text { N30.0 Akute Zystitis } \\
\text { N30.9 Zystistis nicht näher bezeichnet } \\
\text { N39.0 Harnwegsinfektion, Lokalisation nicht näher } \\
\text { bezeichnet }\end{array}$ & $\begin{array}{l}\text { Indizierte Antibiotika aus der Therapeutischen } \\
\text { Hauptgruppe J01 Antibiotika zur systemischen } \\
\text { Anwendung, vgl. - Tab. } 10.10 \mathrm{im} \text { Anhang }\end{array}$ \\
\hline Daten & Ambulante gesicherte Diagnosen & Ambulante Verordnungen \\
\hline \multicolumn{3}{|l|}{ Quelle: WIdO } \\
\hline
\end{tabular}




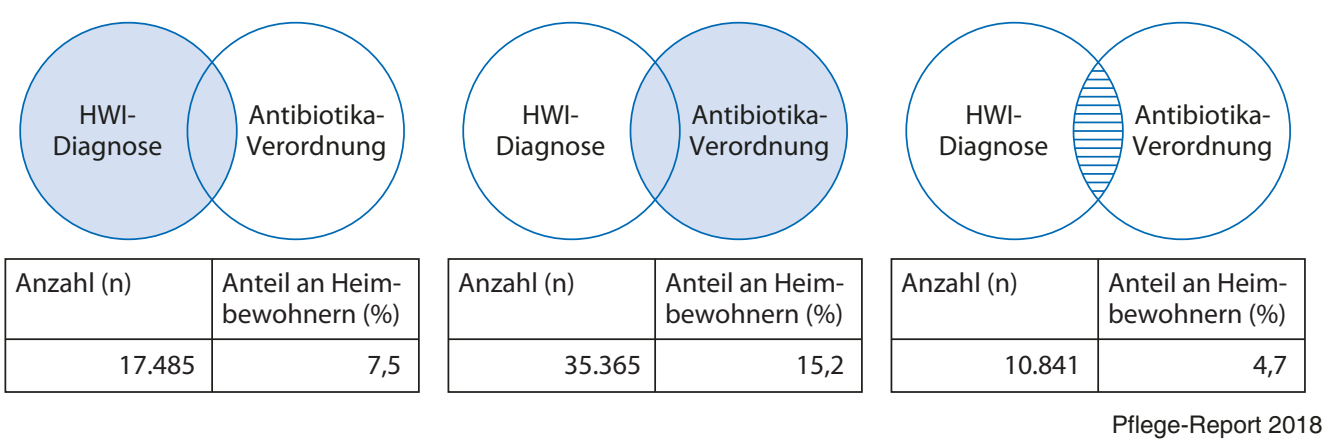

- Abb. 10.2 Häufigkeit von Harnwegsinfekt (HWI)-Diagnosen und Verordnungen von indizierten Antibiotika im Pflegeheim (2015, im Durchschnitt der Quartale) (Quelle: AOK-Daten 2015)

analyse weder die symptomorientierte Validierung der Diagnose möglich noch geht daraus hervor, inwiefern die Antibiotika-Verordnung mit der Harnwegsinfektion assoziiert ist. Die Grundgesamtheit der auf Routinedatenbasis einbezogenen Pflegeheime ist zudem weitaus größer und heterogener freiwillig teilnehmende Einrichtungen wie in der HALT-2-Studie sind i. d. R. auch die "guten Performer" (Selection Bias).

Da die ambulanten Diagnosedaten quartalsbezogen vorliegen, ist es maximal möglich, einen therapeutischen Zusammenhang zwischen Diagnose und Verordnung via Zeitnähe - Diagnose und Verordnung liegen im selben Quartal vor - als Proxy zu schätzen. Ein Ergebnis der HALT-2-Studie war zudem, dass 12,6\% der verabreichten systemischen Antibiotika rein prophylaktisch bei an sich beschwerdefreien Bewohnern zum Einsatz kam (Ruscher et al. 2015).

Die finale Kennzahl (• Tab. 10.6) setzt folglich die Anzahl der (behandelten) HWI-Fälle zur Bewohnerschaft eines Pflegeheims in Relation. Ein HWI gilt als Fall, wenn innerhalb eines Quartals sowohl mindestens eine entsprechende HWI-Diagnose als auch zeitnah, d. h. im gleichen Quartal, mindestens eine Verordnung eines Antibiotikums vorliegt.

\subsubsection{Versorgung mit Antipsychotika im Pflegeheim}

Die Operationalisierung des Antipsychotika-Einsatzes erfolgte mit Hilfe der Wirkstoffgruppe "Antipsychotika« (N05A). Weiterhin testete die Operationalisierung, ob Personen mit Demenz auf Basis von vertragsärztlichen Diagnosen und auf Basis der MDK-Begutachtung zur Pflegebedürftigkeit konkret der Feststellung einer eingeschränkten Alltagskompetenz (EA) im Sinne des $\$ 45$ a SGB XI (vor PSG II) - identifiziert werden können. Der Hilfeund Betreuungsbedarf von Menschen mit demenzbedingten Fähigkeitsstörungen, mit geistigen Behinderungen oder psychischen Erkrankungen wurde bis zur Einführung des neuen Pflegebedürftigkeitsbegriffs im Jahr 2017 zusätzlich zur eigentlichen Einstufung in die Pflegestufen gesondert geregelt und unter einer eingeschränkten Alltagskompetenz erfasst. ${ }^{6}$ Demzufolge ist zum einen EA nicht mit Demenz gleichzusetzen, zum anderen erfüllen Personen mit beginnender bzw. leichter Demenz nicht zwangsläufig die Kriterien für eine EA. Angesichts der hier betrachteten Pflegeheimpopulation im Alter von mindestens 60 Jahren sind diesbezüglich jedoch hohe Übereinstimmungen zu erwarten.

6 Siehe hierzu die (letzte) vor Einführung des neuen Pflegebedürftigkeitsbegriff gültige Richtlinie des GKV-Spitzenverbandes zur Begutachtung von Pflegebedürftigkeit nach dem XI. Buch des Sozialgesetzbuchs (Begutachtungs-Richtlinien - BRi) vom 08.06.2009, geändert durch Beschluss vom 16.04.2013. 

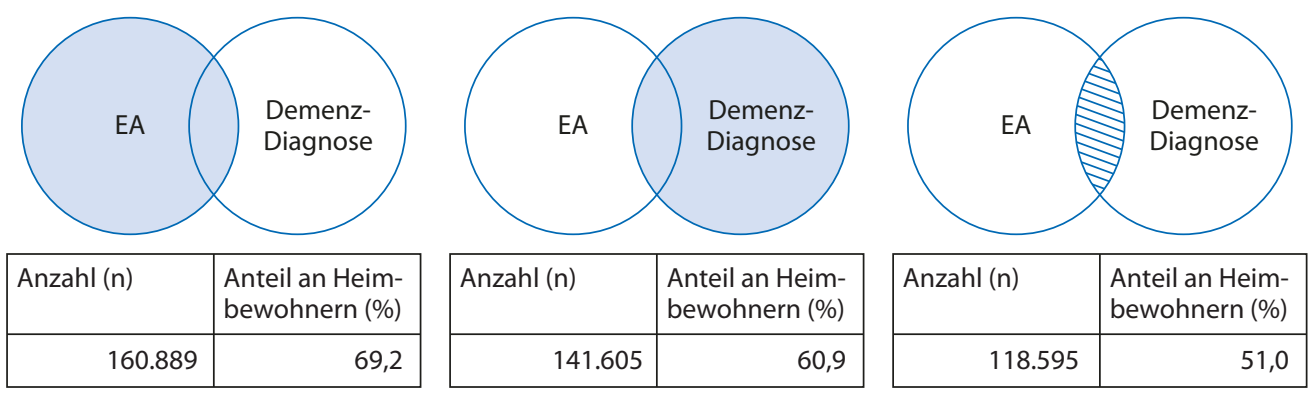

Pflege-Report 2018

- Abb. 10.3 Häufigkeit von Demenz-Diagnosen und eingeschränkter Alltagskompetenz (EA) (2015, im Durchschnitt der Quartale) (Quelle: AOK-Daten 2015)

Die in - Abb. 10.3 dargestellte Häufigkeit von Diagnosen mit Hilfe der EA-Einstufung wirft folglich Fragen auf: Für etwas mehr als die Hälfte der Pflegeheimbewohner $(51,0)$ fand sich im Durchschnitt der Quartale in den Routinedaten sowohl eine EA als auch eine Demenzdiagnose. Lediglich $73,7 \%$ - also gerade einmal drei von vier - der Pflegeheimbewohner (ab 60 Jahre) mit eingeschränkter Alltagskompetenz (EA) hatten eine DemenzDiagnose (•Tab. 10.4). Dass EA nicht mit Demenz gleichzusetzen ist, lässt sich zunächst rein rechtlich

\begin{tabular}{|c|c|}
\hline & ICD-10: Demenz \\
\hline F00 & Demenz bei Alzheimer-Krankheit \\
\hline F01 & Vaskuläre Demenz \\
\hline F02 & $\begin{array}{l}\text { Demenz bei anderenorts klassifizierten } \\
\text { Krankheiten }\end{array}$ \\
\hline F03 & Nicht näher bezeichnete Demenz \\
\hline F051 & Delir bei Demenz \\
\hline G231 & $\begin{array}{l}\text { Progressive supranukleäre Ophthalmoplegie } \\
\text { [Steele-Richardson-Olszewski-Syndrom] }\end{array}$ \\
\hline G30 & Alzheimer-Krankheit \\
\hline G310 & Umschriebene Hirnatrophie \\
\hline G3182 & Lewy-Körper-Demenz \\
\hline $\begin{array}{l}\text { Quelle: } \\
2012\end{array}$ & O in Anlehnung an Schulz und Doblhammer \\
\hline
\end{tabular}

Pflege-Report 2018 begründen. Die Richtlinie zur Feststellung von Personen mit erheblich eingeschränkter Alltagskompetenz und zur Bewertung des Hilfebedarfs zählt neben demenzbedingten Beeinträchtigungen auch dauerhafte Einschränkungen der Alltagskompetenz bei zugrundeliegenden geistigen Behinderungen und psychischen Erkrankungen zu den Gründen für eine EA (GKV-SV 2010).

Andersherum wiesen lediglich 83,8 \% der Personen mit einer Demenz-Diagnose auch eine anerkannte eingeschränkte Alltagskompetenz auf (- Abb. 10.3). Vor diesem Hintergrund wurde bei den Analysen auf die EA-Einstufung des MDK aufgesetzt, da diese - anders als die vertragsärztlich dokumentierten Diagnosen - ausführlichen Regelungen unterworfen ist. Hierfür sprach auch, dass 40,6\% (• Tab. 10.8) der so definierten dementiell erkrankten Bewohner innerhalb eines Quartals eine entsprechende Verordnung haben, was im Prävalenzbereich der epidemiologischen Studien liegt (vgl. $>$ Abschn. 10.2.3).

Die finale Kennzahl (• Tab. 10.6) erfasst somit im Zähler die Summe aller Pflegeheimbewohner, die zu Beginn des Quartals eine EA und mindestens eine Antipsychotikaverordnung im gleichen Quartal aufwiesen. Über das gesamte Jahr können somit maximal vier Fälle (d. h. entsprechende Verordnungen) erfasst werden. Der Nenner der Kennzahl wird analog zur Kennzahl »Dekubitusfälle« ( Abschn. 10.3.2) berechnet. Ebenso wie der Zähler berücksichtigt der Nenner ausschließlich Pflegeheimbewohner mit EA zu Beginn des Quartals. 


\subsection{5 Ärztliche Versorgung im Pflegeheim}

Für die Berücksichtigung der ärztlichen Versorgung von Bewohnern wurden zwei Aspekte gewählt: Angesichts des gesetzlich formulierten Ziels einer über den Hausarzt koordinierten ambulanten Versorgung mit festen Ansprechpartnern in der stationären Langzeitpflege wird die Zahl der Hausärzte mit Kontakt zum jeweiligen Pflegeheim gemessen. Die Kennzahl zur hausärztlichen Versorgung erfasst die Anzahl der Hausärzte je 100 Bewohner eines
Pflegeheims innerhalb eines Jahres (• Tab. 10.6). Die Operationalisierung erfolgte mit Hilfe der vertragsärztlichen Abrechnungsinformationen als Resultat der Leistungserbringung des Hausarztes. Gezählt wurde der Arzt unabhängig davon, ob der Pflegeheimbewohner die Praxis des Arztes aufsuchte oder ob es sich um eine Arztvisite im Pflegeheim handelte.

Die oben aufgeführten Versorgungsdefizite im Bereich der Diagnostik und Therapie von Demenz und Depressionen wie auch die Fehlversorgung mit Psychopharmaka begründen ferner die Analyse zur

- Tab. 10.5 Ambulant-sensitive Hospitalisierungen - relevante Klassifikationen zur Identifizierung auf Routinedatenbasis

\begin{tabular}{|c|c|}
\hline Krankheitsgruppe (Kerngruppe) & ICD-Codes \\
\hline Infektiöse Darmkrankheiten & $\mathrm{A} 01, \mathrm{A02}, \mathrm{A04}, \mathrm{A05}, \mathrm{A} 07-\mathrm{A} 09$ \\
\hline Krankheiten der Haut, Unterhaut und Hautanhangsgebilde & A46, L01, L02, L04, L08.0, L08.8, L08.9, L60.0, L72.1, L98.0 \\
\hline Mangelerkrankungen und Thalassämie & D50, D51, D52, D53.1, D56, E40-E64, R63.6 \\
\hline Diabetes mellitus & E10.2-E10.8, E10.9, E11, E13.6, E13.7, E13.9, E14, E16.2 \\
\hline $\begin{array}{l}\text { Psychische und Verhaltensstörungen durch Alkohol oder } \\
\text { Opioide }\end{array}$ & F10, F11 \\
\hline Depressionen & F32, F33 \\
\hline (Andere) Psychische und Verhaltensstörungen & $F 40, F 41, F 43, F 45, F 50.0, F 50.2, F 60$ \\
\hline Schlafstörungen & G47 \\
\hline Erkrankungen der Weichteile und peripherer Nerven & G56.0, M67.4, M71.3, M75-M77, M79 \\
\hline Altersbedingter grauer Star und grüner Star (Glaukom) & $\mathrm{H} 25, \mathrm{H} 40$ \\
\hline HNO-Infektionen & H66, J01-J03, J06, J31, J32, J35 \\
\hline Andere Krankheiten des Kreislaufsystems & $\begin{array}{l}105,106,108.0,149.8,149.9,167.2,167.4,170,173,178,180.0 \\
180.80,183,186,187,195, \text { R00.0, R00.2, R47.0 }\end{array}$ \\
\hline Bluthochdruck und Folgeerkrankungen & $110-115$ \\
\hline Ischämische Herzkrankheiten & $120,125.0,125.1,125.5,125.6,125.8,125.9$ \\
\hline Herzinsuffizienz & 150 \\
\hline Grippe und Pneumonie & $\begin{array}{l}\mathrm{J} 10, \mathrm{~J} 11, \mathrm{~J} 13, \mathrm{~J} 14, \mathrm{~J} 15.3, \mathrm{~J} 15.4, \mathrm{~J} 15.7, \mathrm{~J} 15.8, \mathrm{~J} 15.9, \mathrm{~J} 16.8, \\
\mathrm{~J} 18.0, \mathrm{~J} 18.1, \mathrm{~J} 18.8, \mathrm{~J} 18.9\end{array}$ \\
\hline Bronchitis, COPD und Bronchiektasie & $\mathrm{J} 20, \mathrm{~J} 21, \mathrm{~J} 40-\mathrm{J} 44, \mathrm{~J} 47$ \\
\hline $\begin{array}{l}\text { Krankheiten der Zähne und des Zahnhalteapparates } \\
\text { sowie der Lippe und der Mundschleimhaut }\end{array}$ & $\mathrm{K} 02, \mathrm{~K} 04-\mathrm{K} 06, \mathrm{~K} 08, \mathrm{~K} 12, \mathrm{~K} 13$ \\
\hline Nicht-infektiöse Krankheiten des Magen-Darm-Trakts & K52.2, K52.8, K52.9, K57, K58, K59.0 \\
\hline Kniegelenksarthrose & M17.0, M17.1, M17.4, M17.5, M17.9 \\
\hline Rückenschmerzen, Dorsopathien & M42, M47, M53, M54 \\
\hline Krankheiten des Harnsystems & N30, N34, N39.0 \\
\hline Quelle: WIdO nach Sundmacher und Schüttig 2015 & \\
\hline
\end{tabular}


Inanspruchnahme von Neurologen, Nervenärzten oder Psychiatern. Die Kennzahl zur Versorgung durch diese Facharztgruppen betrachtet analog zur Antipsychotikaverordnung ( Abschn. 10.3.4) ausschließlich Pflegeheimbewohner mit EA. Fälle beim Neurologen, Nervenarzt oder Psychiater werden je Quartal berechnet, d. h. Pflegeheimbewohner mit anerkannter EA im Sinne des SGB XI mit mindestens einem Arztkontakt im Quartal bei einer der in - Tab. 10.6 gelisteten Fachgruppen sind »ein Fall«. Dabei war irrelevant, ob der Bewohner den Facharzt aufsuchte oder umgekehrt. Die Referenzpopulation wird für die Betrachtung der hausärztlichen Versorgung wiederum analog zur Kennzahl »Dekubitusfälle« ( $\triangleright$ Abschn. 10.3.2) definiert und ebenfalls auf Pflegeheimbewohner mit EA eingeschränkt.

\subsubsection{Ambulant-sensitive Hospitalisierungen von Pflegeheimbewohnern}

Tab. 10.5 listet alle 22 ambulant-sensitiven Krankheitsgruppen auf, die bei der Operationalisierung der Kennzahl ASK je 100 Bewohner pro Pflegeheim und Jahr Berücksichtigung fanden. Es handelt sich dabei um die Kernindikationsgruppen des deutschen ASK-Katalogs, denen insgesamt 163 Diagnosen zugeordnet sind. Betrachtet werden dabei ausschließlich die Hauptdiagnosen (stationäre Entlassungsdiagnosen), da diese gemäß Kodierrichtlinien im DRG-System den Krankenhausaufenthalt veranlassten (DKG et al. 2017).

Analog zum Vorgehen bei den anderen hier vorgestellten Kennzahlen konkretisierten auch im Falle der ASK zunächst explorative Analysen die Algorithmen der finalen Operationalisierung. Sie ergaben, dass im Durchschnitt der Quartale 19,8\% aller betrachteten Bewohner im Jahr 2015 im Krankenhaus versorgt wurden - 40,5\% dieser Bewohner mit Hospitalisierungen (d. h. 8,0 \% aller Pflegeheimbewohner) waren im Durchschnitt der Quartale im Jahr 2015 definitionsgemäß nach Sundmacher et al. (2015) Patienten mit mindestens einem ASK (• Abb. 10.4). Ein Vergleich der ASK-Häufigkeit mit Erkenntnissen aus anderen Ländern (da für den deutschen Kontext keine Informationen zu ASK im stationären Pflegesetting $\mathrm{zu}$ finden sind) ist nur

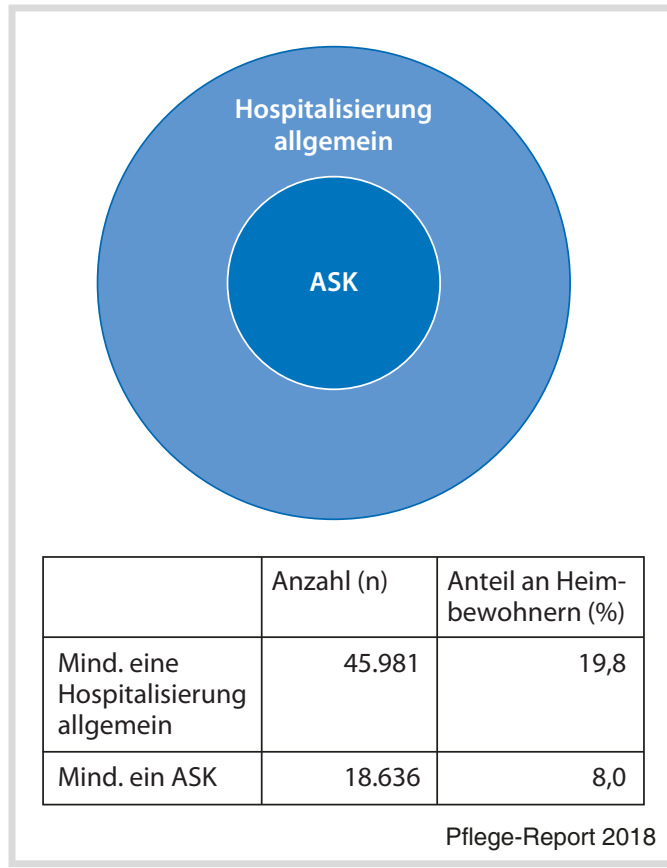

- Abb. 10.4 Häufigkeit von ambulant-sensitiven Krankenhausfällen (ASK) im Pflegeheim (2015, im Durchschnitt der Quartale) (Quelle: AOK-Daten 2015)

eingeschränkt möglich. Neben den variierenden Gesundheits- und Pflegesystemen unterscheiden sich nicht zuletzt die ASK-Kataloge und die Klassifikationsbasis selbst. Dem kanadischen Health Quality Ontario Institut (HQO) zufolge waren ein Drittel der Pflegeheimbewohner in der Region Ontario, Alberta und Yukon durchschnittlich einmal jährlich in einer Notaufnahme - ein Drittel von ihnen galt als potenziell vermeidbar (HQO 2016).

Um zu erfahren, welche Erkrankungen die ASK bei Pflegeheimbewohnern begründen, fokussierte eine Detailanalyse die ASK-Diagnosen im ersten Quartal 2015 ( $\mathrm{n}=22.754)$. - Abb. 10.5 listet die häufigsten ASK-Diagnosen auf. An erster Stelle steht die Diagnose Herzinsuffizienz (17,6 \%), gefolgt von der sonstigen chronischen obstruktiven Lungenkrankheit mit 7,9\% und der akuten Bronchitis mit 7,3\%. Auch Beschwerdebilder des Harnsystems sowie des metabolischen Systems gehören zu den abgerechneten ASK-Diagnosen.

Im Resultat der Operationalisierung deutet die heimindividuelle ASK-Rate als Kennzahl zur Mes- 


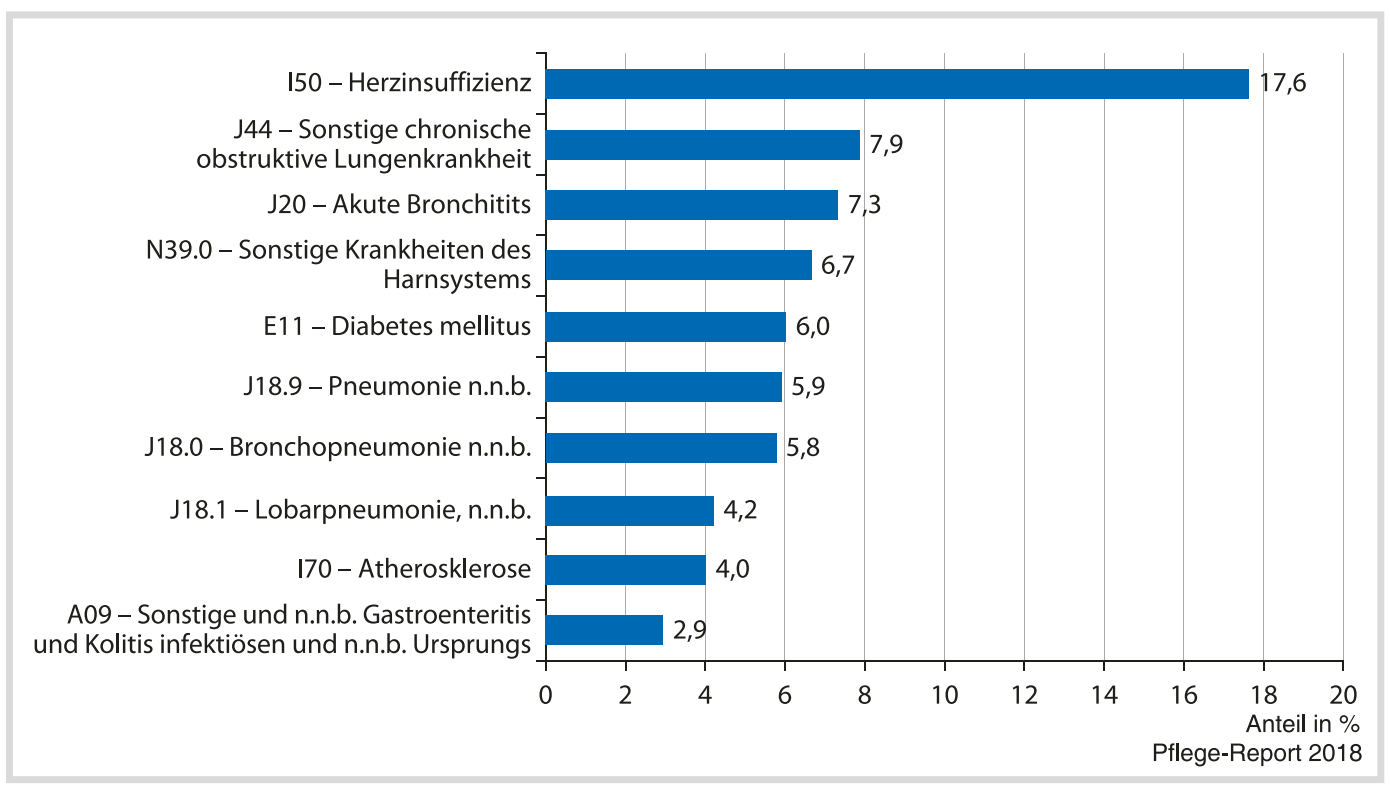

- Abb. 10.5 Verteilung der zehn häufigsten Hauptdiagnosen ambulant-sensitiver Hospitalisierungen im Pflegeheim in \% (im ersten Quartal 2015) (Quelle: AOK-Daten 2015)

sung der Ergebnisqualität in der stationären Langzeitpflege auf folgende Merkmale hin (• Tab. 10.6): Ein ASK liegt immer dann vor, wenn eine der Diagnosen des von Sundmacher et al. (2015) definierten ASK-Katalogs (Kerngruppe) als Hauptdiagnose (Entlassungsdiagnose) einen vollstationären Krankenhausaufenthalt des Bewohners im Referenzjahr begründet. Anders als bei den bisherigen Kennzahlen kann ein Bewohner bei dieser Kennzahl rechnerisch mehr als vier ASK-Fälle im Jahr aufweisen. Die Referenzpopulation, auf die sich die erfassten ASK-Fälle beziehen, wird analog zur Operationalisierung der Kennzahl »Dekubitusfälle« gefasst.

\subsection{Ergebnisse}

Im Referenzjahr 2015 trat im Durchschnitt der Quartale bei 2,1 \% der Bewohner ein neuer Dekubitus auf (•Tab. 10.8). Nach der hier gewählten Operationalisierung der Kennzahl (• Tab. 10.6) treten im Mittel 8,5 Dekubitusfälle je 100 Bewohner in Pflegeheimen neu auf (•Tab. 10.7). • Abb. 10.6 veranschaulicht, in welchem Umfang die Qualitätskennzahl zwischen den Pflegeheimen variiert: Beim auffälligsten Viertel der Einrichtungen finden sich 11,9 oder mehr Dekubitusfälle je 100 Bewohner, bei dem Viertel mit den niedrigsten Raten hingegen lediglich 4,0 oder weniger Fälle. Das auffälligste Viertel der Pflegeheime hat damit mehr als dreimal so viele Dekubitusfälle wie jenes mit den niedrigsten Raten (Grenze des 75. Perzentil im Verhältnis zur Grenze des 25. Perzentil = 2,9). Bei $5 \%$ der Pflegeheime (95. Perzentil) betrug die Rate sogar 19,8 Fälle oder mehr je 100 Bewohner. Mit Blick auf die bestehenden Standards für Prävention und Versorgung und die darauf basierende Einschätzung, dass das Auftreten eines Dekubitus weitgehend verhindert werden kann, liefern diese Ergebnisse somit einen wichtigen Hinweis auf die erhebliche Variation der Versorgungsqualität zwischen den Pflegeheimen.

Bei der Kennzahl Harnwegsinfekte (HWI) zeigt sich ein ähnliches Bild: Im Durchschnitt der Quartale haben 4,7\% der Bewohner nach der hier gewählten Operationalisierung (• Tab. 10.6) einen HWI (•Tab. 10.8). Das Wertespektrum der HWIFälle je 100 Bewohner und Jahr erstreckt sich von Null bis zum Maximalwert von 105,7 Infektionen; in $5 \%$ der Pflegeheime werden 44,6 oder mehr Harnwegsinfekte je 100 Bewohnerjahre erfasst. Für 


\begin{tabular}{|c|c|c|c|c|c|c|c|}
\hline & 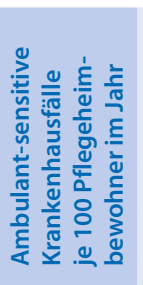 & 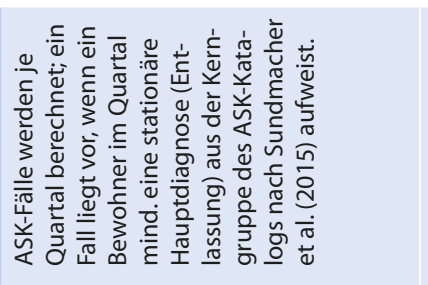 & 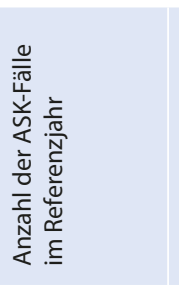 & 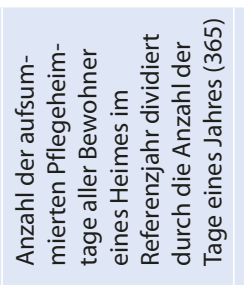 & 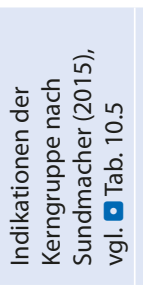 & 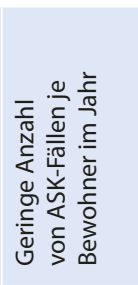 & \\
\hline & 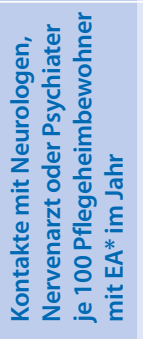 & 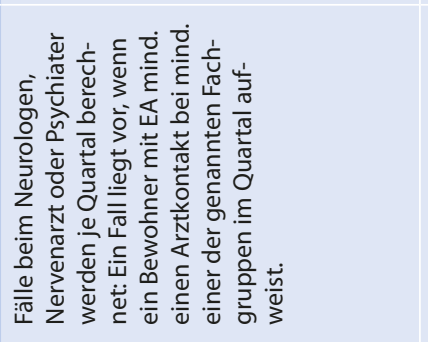 & 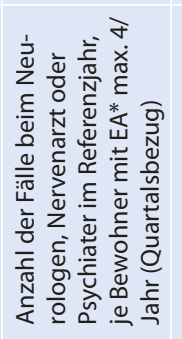 & 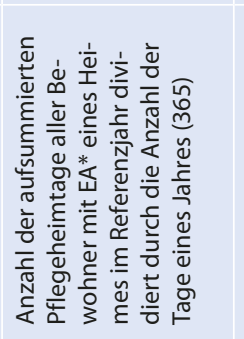 & 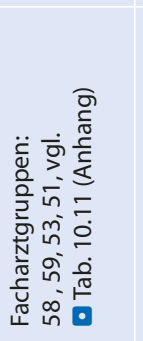 & 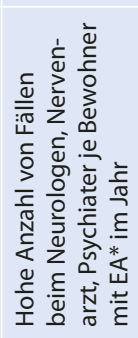 & \\
\hline $\begin{array}{l}\frac{0}{0} \\
\frac{5}{0}\end{array}$ & 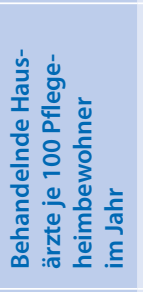 & 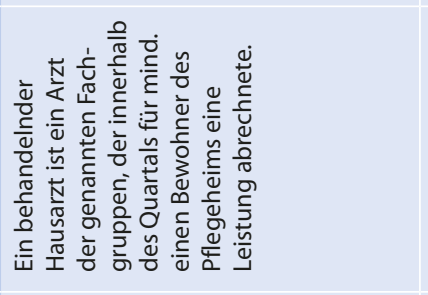 & 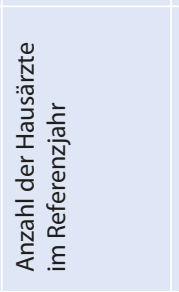 & 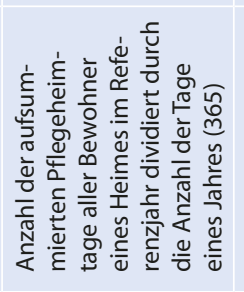 & 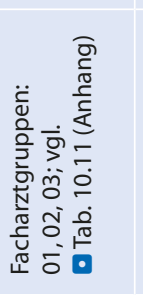 & 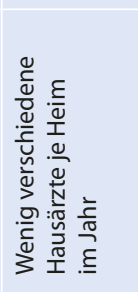 & \\
\hline 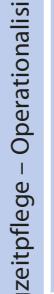 & 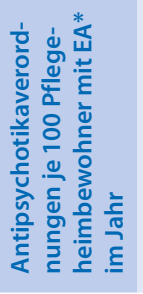 & 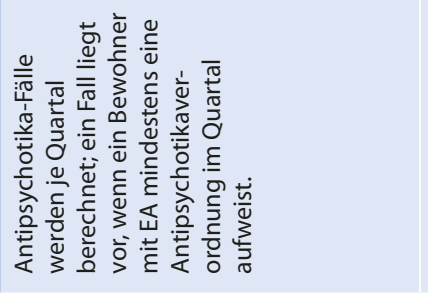 & 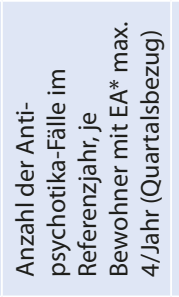 & 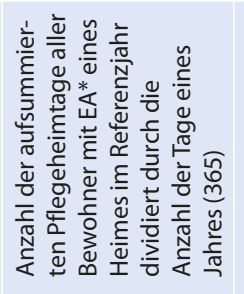 & 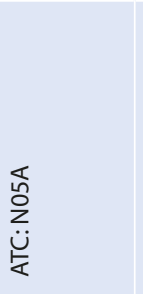 & 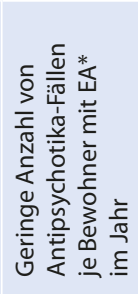 & \\
\hline 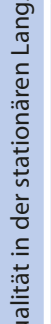 & 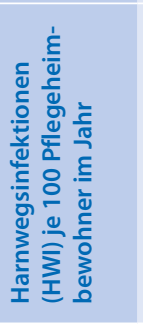 & 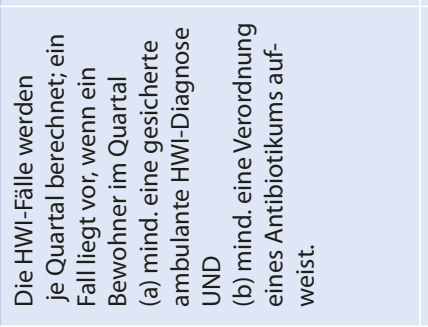 & 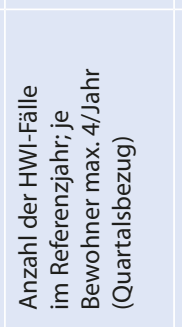 & 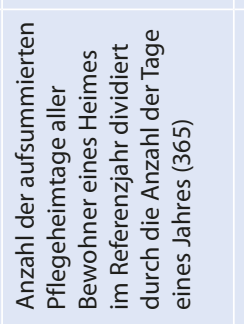 & 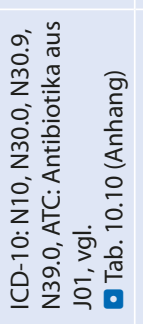 & 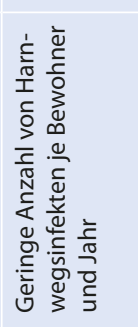 & \\
\hline 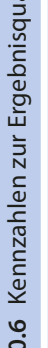 & 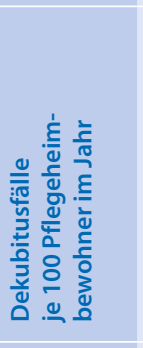 & 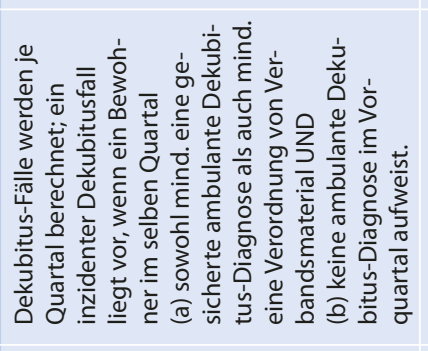 & 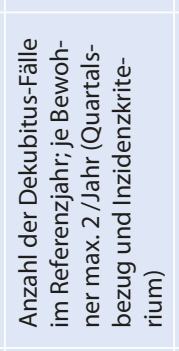 & 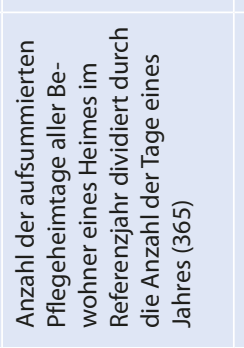 & 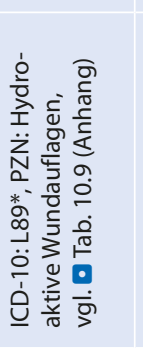 & 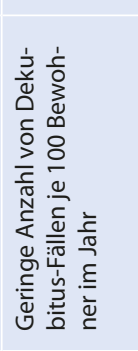 & 응 \\
\hline$\frac{0}{\circ}$ & & $\overline{\overline{\widetilde{\Gamma}}}$ & $\frac{\frac{\bar{d}}{\frac{\pi}{i n}}}{\frac{N}{N}}$ & 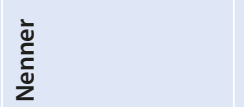 & $\begin{array}{l}\underline{y} \\
\text { ö } \\
\underline{g}\end{array}$ & $\underset{\substack{\mathbf{v} \\
d \\
d}}{ }$ & 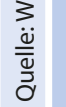 \\
\hline
\end{tabular}




\begin{tabular}{|c|c|c|c|c|c|c|}
\hline & \multicolumn{6}{|c|}{ Operationalisierte Kennzahlfälle je 100 Bewohner im Jahr 2015} \\
\hline & $\begin{array}{l}\text { Anzahl Fälle } \\
\text { neu aufge- } \\
\text { tretener } \\
\text { Dekubiti }\end{array}$ & $\begin{array}{l}\text { Anzahl Fälle } \\
\text { (behandelter) } \\
\text { Harnwegs- } \\
\text { infektionen }\end{array}$ & $\begin{array}{l}\text { Anzahl Anti- } \\
\text { psychotika- } \\
\text { fälle bezogen } \\
\text { auf Bewohner } \\
\text { mit EA* }\end{array}$ & $\begin{array}{l}\text { Anzahl der } \\
\text { behan- } \\
\text { delnden } \\
\text { Hausärzte }\end{array}$ & $\begin{array}{l}\text { Anzahl Neurolo- } \\
\text { gen-, Nervenarzt- } \\
\text { oder Psychiater- } \\
\text { Fälle bezogen } \\
\text { auf Bewohner } \\
\text { mit EA* }\end{array}$ & $\begin{array}{l}\text { Anzahl ambu- } \\
\text { lant-sensitiv } \\
\text { eingestufter } \\
\text { Hospitalisie- } \\
\text { rungen }\end{array}$ \\
\hline Pflegeheime & 5.622 & 5.622 & 5.622 & 5.622 & 5.622 & 5.622 \\
\hline $\begin{array}{l}\text { Bewohner Refe- } \\
\text { renzpopulation } \\
(\text { Nenner)** }\end{array}$ & 229.881 & 229.881 & 159.103 & 229.881 & 159.103 & 229.881 \\
\hline Fälle (n, Zähler) & 19.473 & 43.363 & 261.046 & 134.517 & 284.416 & 74.542 \\
\hline Minimum & 0,0 & 0,0 & 9,3 & 2,0 & 0,0 & 0,0 \\
\hline 25. Perzentil & 4,0 & 10,1 & 131,9 & 40,6 & 44,8 & 21,6 \\
\hline Median & 7,8 & 17,4 & 161,8 & 58,3 & 175,1 & 30,6 \\
\hline 75. Perzentil & 11,9 & 26,1 & 194,4 & 81,4 & 279,4 & 41,6 \\
\hline 95. Perzentil & 19,8 & 44,6 & 249,4 & 124,5 & 368,6 & 62,5 \\
\hline Maximum & 43,5 & 105,7 & 403,5 & 218,7 & 408,1 & 119,6 \\
\hline $\begin{array}{l}\text { Mittelwert über } \\
\text { alle Pflegeheime }\end{array}$ & 8,5 & 18,9 & 164,1 & 58,5 & 178,8 & 32,4 \\
\hline $\begin{array}{l}\text { Standard- } \\
\text { abweichung }\end{array}$ & 6,0 & 13,4 & 48,3 & 31,5 & 124,3 & 16,1 \\
\hline 75./25. Perzentil & 2,9 & 2,6 & 1,5 & 2,0 & 6,2 & 1,9 \\
\hline
\end{tabular}

Quelle: AOK-Daten 2015

ein Viertel der Pflegeheime (75. Perzentil) ergab sich mit mindestens 26,1 Fällen pro 100 Bewohner eine zweieinhalb mal so hohe Rate wie bei jenen 25 \% der Pflegeheime mit den niedrigsten Raten mit maximal 10,1 Fällen (• Tab. 10.7 und $\bullet$ Abb. 10.6). Unter der Annahme, dass es sich bei dieser Infektionserkrankung um einen »Parameter der hygienischen Ergebnisqualität « (Engelhart et al. 2009) im Pflegeheim handelt, ist die beobachtete Variation der HWI-Fälle im Pflegeheim als kritisches Ergebnis für die Versorgungsqualität zu verstehen und sollte weitergehend analysiert werden.

Die Ergebnisse zu den Antipsychotikaverordnungen je 100 Pflegeheimbewohner mit eingeschränkter Alltagskompetenz (EA) im Referenzjahr unterstreichen die Bedeutung dieser Kenn- zahl (•Tab. 10.7 und • Abb. 10.6): Im Durchschnitt der Quartale hatten 40,6\% aller Bewohner mit EA mind. eine Antipsychotikaverordnung (•Tab. 10.8). Im Sinne der operationalisierten Kennzahl traten im auffälligsten Viertel der Pflegeheime (ab 75. Perzentil) 194,4 und mehr Antipsychotika-Fälle auf, d. h. dass in einem von vier Pflegeheimen durchschnittlich jeder Bewohner mit EA zumindest in knapp zwei Quartalen eine Antipsychotikaverordnung erhielt (• Tab. 10.7). Auffällig an dieser Kennzahl ist ferner, dass mit Blick auf den empfohlenen restriktiven Einsatz dieser Wirkstoffe auch in den Pflegeheimen mit den niedrigsten Raten (bis zum 25. Perzentil) bereits bis zu 131,9 Antipsychotika-Fälle pro 100 Bewohner und Jahr auftreten. 


\begin{tabular}{l|l|l|}
\hline - Tab. 10.8 Anteil Bewohner, die je Quartal mindestens einmal von einem im Sinne der Kennzahl operationalisierten \\
\begin{tabular}{l} 
Zielereignis betroffen waren, im Durchschnitt der Quartale (2015) )(*EA - eingeschränkte Alltagskompetenz) \\
\hline Anteil Bewohner mit neu aufgetretenem Dekubitus
\end{tabular} & 2,1 \\
\hline Anteil Bewohner mit behandelter Harnwegsinfektion & 4,7 \\
\hline Anteil Bewohner mit mindestens einer Antipsychotikaverordnung, bezogen auf Bewohner mit EA* & 40,6 \\
\hline $\begin{array}{l}\text { Anteil Bewohner mit mindestens einem Kontakt zu einem Neurologen, Nervenarzt oder Psychiater, } \\
\text { bezogen auf Bewohner mit EA* }\end{array}$ & 30,6 \\
\hline Anteil Bewohner mit mindestens einer ambulant-sensitiv eingestuften Hospitalisierung & 8,0 \\
\hline Quelle: AOK-Daten 2015 &
\end{tabular}

Pflege-Report 2015
Die Zahl der Fälle in Pflegeheimen ab dem 75. Perzentil der Verteilung liegen mit 195 Fällen und mehr um mindestens das 1,5-Fache höher, die $5 \%$ auffälligsten Einrichtungen (ab 95. Perzentil) mit rund 250 und mehr knapp zweimal so hoch wie im am wenigsten auffälligen Viertel (bis 25. Perzentil). Diese Ergebnisse bestätigen folglich die Befunde aus zahlreichen Studien zur AntipsychotikaFehlversorgung und geben Hinweise darauf, dass die Versorgungsrealität in allen hier betrachteten stationären Pflegeeinrichtungen häufig nicht einer leitliniengerechten Priorisierung nicht-medikamentöser Therapieansätze entspricht. Das Potenzial der Versorgungsoptimierung ist an dieser Stelle folglich erheblich.

Ferner wirft der Blick auf die hausärztliche Versorgung der Pflegeheimbewohner erhebliche Fragen hinsichtlich der effektiven Koordinierung zwischen Arztpraxen und Pflegeheimen auf (• Tab. 10.7 und - Abb. 10.6): Durchschnittlich 58,5 unterschiedliche Hausärzte je 100 Bewohner im Pflegeheim gewährleisten die Hausarztversorgung. Die Ergebnisraten verweisen auf einen eklatant hohen Koordinierungsaufwand für das Pflegepersonal bzw. auf die dahinter zu vermutende defizitäre intraprofessionelle Kommunikation und die damit assoziierten Folgen, wie z. B. Defizite im Bereich Diagnostik und/oder Versorgung oder auch vermeidbare Krankenhauseinweisungen. Ferner verweisen die Ergebnisse wiederum auf eine ausgeprägte Heterogenität der hausärztlichen Versorgung. Beim auffälligsten Viertel der Pflegeheime sind mindestens 81,4 Ärzte je 100 Bewohner involviert, bei $5 \%$ der Pflegeheime sogar 124,5 Ärzte und mehr. Dass mehr Ärzte als Bewohner involviert sind, ist hierbei methodisch erklärbar. Für die Bildung der Referenzpopulation (Nenner) wurde auf »Vollzeit-Bewohner « normiert und damit werden faktisch mehr als 100 (echte) Personen betrachtet, die jeweils beim Einzug ins Pflegeheim ihren eigenen Hausarzt »mitbringen « können (vgl. - Abschn. 10.3.2). Zudem besteht die Möglichkeit, dass Bewohner im Jahresverlauf ihren Hausarzt wechseln oder mehrere unterschiedliche Hausärzte in Anspruch nehmen (z. B. durch Inanspruchnahme eines weiteren Arztes während eines ambulanten notärztlichen Einsatzes).

Mit der Kennzahl zur Anzahl der Kontakte mit Neurologen, Nervenärzten und Psychiatern je 100 Bewohner mit EA (•Tab. 10.7 und • Abb. 10.6) wurde ergänzend - exemplarisch - die Qualität der fachärztlichen Versorgung betrachtet. Im Durchschnitt der Quartale hatte lediglich rund jeder Dritte $(30,6 \%)$ der Bewohner mit EA mindestens einen Kontakt zu einem Vertragsarzt dieser Fachgruppe (• Tab. 10.8). Im Sinne der hier operationalisierten Kennzahl entfielen im Mittel 178,8 derartige Kontakte auf 100 Bewohner mit EA im Jahr, was bedeutet, dass die Betroffenen seltener als zweimal im Jahr (1,8 Kontakte je Bewohner) einen entsprechenden Facharzt sehen (•Tab. 10.7). Bei den Pflegeheimen mit den höchsten Kontaktraten (ab dem 95. Perzentil) zeigen die Analysen hingegen rund 370 entsprechende Arztkontakte je 100 Bewohner mit EA und damit eine fast kontinuierliche Betreuung über alle vier Quartale des Jahres hinweg. Die Pflegeheime mit Raten ab dem 75. Perzentil haben immerhin 


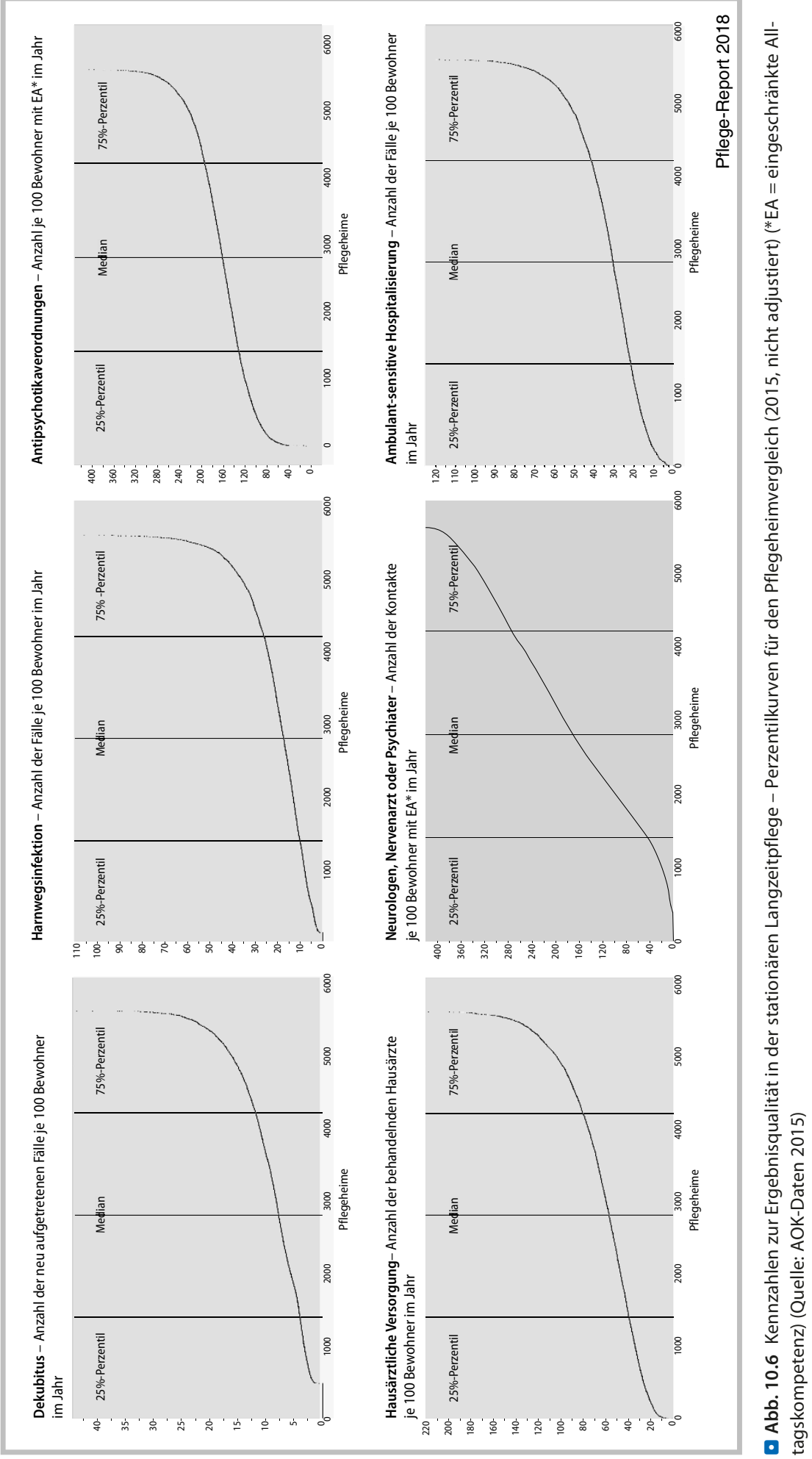


noch mindestens 279,4 Kontakte dieser Art, während beim Viertel mit den niedrigsten Kontaktraten (bis zum 25. Perzentil) mit maximal 44,8 sehr wenige Kontakte auf die gleiche Bewohneranzahl mit EA zu beobachten sind - hier sieht der Betroffene im Mittel den Facharzt einmal alle zwei Jahre. Die Unterschiede zwischen dem Viertel der Pflegeheime mit den höchsten (hier die »besten «) und den niedrigsten (hier die »schlechtesten «) Raten sind bei dieser Kennzahl besonders hoch. Ausgehend von dieser erheblichen Variation der Kennzahl für die neurologische, nervenärztliche und psychiatrische Versorgung und ebenso angesichts des geringen Anteils der Pflegeheimbewohner mit EA, die im Referenzjahr überhaupt mit dieser Fachgruppe in Kontakt kamen, besteht hier Optimierungs-, gepaart mit weitergehendem Analysebedarf.

Auch für den Kontext der ambulant-sensitiven Krankenhausfälle (ASK) zeigte sich das Potenzial für die Optimierung der Versorgung: Pro Quartal wurden 8,0 \% der Pflegeheimbewohner im Jahr 2015 aufgrund einer definitionsgemäß ambulant-sensitiven Indikation ins Krankenhaus eingewiesen (•Tab. 10.8). Im Durchschnitt kommen 32,4 ambulant-sensitive Krankenhausfälle auf 100 Bewohner pro Jahr (- Tab. 10.7). Auch bei dieser Kennzahl haben die Pflegeheime im auffälligen Viertel (ab dem 75. Perzentil) mit 41,6 und mehr Hospitalisierungen mehr als doppelt so hohe Raten wie jene in dieser Hinsicht unauffälligen Pflegeheime (bis zum 25. Perzentil) mit lediglich 21,6 oder weniger ambulant-sensitiven Krankenhausfällen (•Tab. 10.7 und $\bullet$ Abb. 10.6). In jenen $5 \%$ der Einrichtungen mit den höchsten Raten (ab dem 95. Perzentil) entfielen 62,5 oder mehr ASK auf 100 Bewohner: Zwei von drei Bewohnern wurden demzufolge im Jahr 2015 mit ambulant-sensitiver Diagnose hospitalisiert bzw. wenige Bewohner mehrfach. Der Grundthese des ASK-Konzepts zufolge handelt es sich bei diesen Krankenhausaufenthalten mit hoher Wahrscheinlichkeit um vermeidbare Behandlungen, die für Bewohner unnötige Risiken für die somatische und psychische Gesundheit bedeuten. Auch wenn nicht alle dieser definitorisch "vermeidbaren « Fälle real vermeidbar sind, wirft dieses Ergebnis der starken Variation zwischen den Pflegeheimen wichtige Fragen für weitergehende Analysen zur Qualität und Steuerung der - insbesondere (fach-) ärztlichen - Versorgung im Pflegeheim auf.

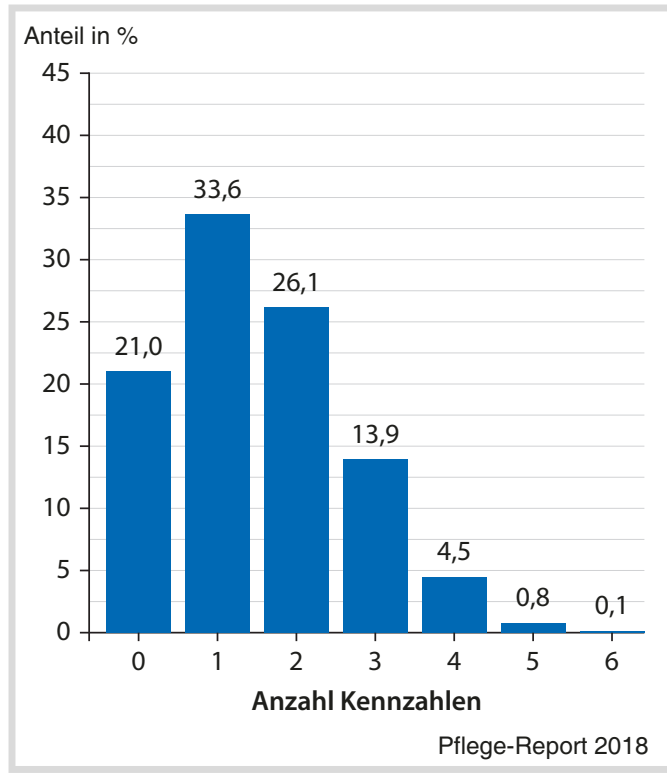

- Abb. 10.7 Pflegeheime mit Auffälligkeiten bei mehreren Kennzahlen - Anteil in \% (2015, nicht adjustiert) (Quelle: AOK-Daten 2015)

Jede einzelne der operationalisierten Kennzahlen gibt damit Hinweise auf eine erhebliche Schwankung der Versorgungsqualität zwischen den Pflegeheimen. Um zu eruieren, ob sich die beobachteten Defizite bei spezifischen Pflegeheimen verdichten, wurden in einer zusätzlichen Analyse die Anzahl der auffälligen Kennwerte auf Pflegeheimebene ermittelt. Als auffällig wurden dabei Ergebnisse mit Werten ab dem 75. Perzentil eingestuft; mit Ausnahme der Kennzahl zur fachärztlichen Versorgung dort wurden aufgrund der umgekehrten Zielrichtung Werte ab dem 25. Perzentil als auffällig markiert. A Abb. 10.7 zeigt zwei markante Ergebnisse: Mit einem Anteil von 19,3 \% wies fast ein Fünftel aller Pflegeheime für drei und mehr der hier betrachteten sechs Kennzahlen Auffälligkeiten auf. Lediglich 21,0 \% der Pflegeheime lagen demgegenüber bei keiner der sechs Kennzahlen im Bereich des »auffälligsten Viertels«. Bei einem Viertel (26,1\%) waren zwei Kennzahlen auffällig. Perspektivisch können die betrachteten routinedatenbasierten Kennzahlen folglich genutzt werden, gezielt Pflegeheime zu adressieren, bei denen sich die Hinweise auf Defizite verdichten. 


\subsection{Diskussion}

Im vorliegenden Beitrag wurden insgesamt sechs Kennzahlen zur Qualität in der stationären Langzeitpflege auf Routinedatenbasis entwickelt und diese einem ersten empirischen Test unterzogen. Aus diesen Analysen ergeben sich zwei grundlegende Erkenntnisse: Erstens: Die Operationalisierung von Kennzahlen auf Basis der in den Routinedaten enthaltenen versichertenbezogenen Merkmale zur Messung der Outcome-Qualität ist realisierbar. Die Vergleiche der explorativen Analysen im Zuge der Operationalisierung $(\checkmark$ Abschn. 10.3$)$ passten - mit Ausnahme der HWI-Kennzahl - zu den gesichteten Befunden aus epidemiologischen Prävalenzstudien ${ }^{7}$. Bereits im jetzigen Stadium lassen sich aus den Ergebnissen wertvolle Schlüsse für die Versorgungsqualität in deutschen Pflegeheimen ziehen. Basierend auf Daten zu mehr als der Hälfte aller stationären Pflegeeinrichtungen stellte die erstmals in Deutschland durchgeführte Analyse eine starke Variation der Outcomes in den ausgewählten Versorgungsaspekten fest. Dies betraf jede Kennzahl mit Ausnahme des dementiell bedingten Antipsychotika-Einsatzes, bei dem nicht in erster Linie die Variation, sondern primär der Umfang der Verordnungshäufigkeit selbst ein relevanter Hinweis auf eine erforderliche Optimierung der pflegerischen und ärztlichen Versorgung ist. Erhebliche Unterschiede in der Versorgungsqualität lassen sich auch aus dem gehäuften Auftreten von gleich mehreren auffälligen Kennzahlraten je Pflegeheim ableiten.

Um die Aussagekraft der entwickelten Kennzahlen weiter abzusichern - dies ist die zweite Erkenntnis -, verbleiben verschiedene methodischkonzeptionelle Herausforderungen. Dies betrifft zum einen die Validierung der Aufgreifkriterien. Zur Abweichung der diagnostizierten von der tatsächlichen Morbidität in der Bezugspopulation

7 Die Forschungslage im Bereich qualitätsrelevanter Outcomeforschung in der stationären Langzeitpflege ist von einer hohen Heterogenität der Studiendesigns und Ergebnisse gekennzeichnet (Burston et al. 2014). Die Aufarbeitung von vergleichbaren Daten aus der aktuellen Forschungslandschaft zu intensivieren ist somit eine der Aufgaben für künftige Indikatorentwicklungen auf Routinedatenbasis. kommt bei Routinedatenanalysen die vertragsärztliche Abrechnungslogik hinzu und limitiert die Güte einer quasi-epidemiologischen Schätzung zusätzlich. Eine Unter- bzw. Übererfassung von Diagnosen durch die Leistungserbringer kann dann leicht zu einer Unter- bzw. Überschätzung der Erkrankungshäufigkeit (IGES 2012) führen. Vor diesem Hintergrund bezog der vorliegende Beitrag therapeutische Leistungsziffern (Verbandsmaterial zur Wundversorgung bzw. Antibiotika-Verordnung) für die Identifizierung von Dekubitus-Fällen und Harnwegsinfekten ein (interne Validierung). Daraus leitet sich der Bedarf einer weiteren Spezifizierung der Algorithmen ab: Erkrankungsfälle, aufgegriffen durch Diagnose in Kombination mit therapeutischer Verordnung, können auch als (positiver) Hinweis gewertet werden, dass eine Versorgung in dem jeweiligen Pflegeheim überhaupt stattfindet. Demgegenüber würden Pflegeheime ohne (adäquate) Versorgung dieser Erkrankungen (d. h. ohne Verordnungen von Verbandsmaterial bzw. Antibiotika bei dokumentierter Diagnose) anscheinend keine Fälle aufweisen.

Ferner ergeben sich aufgrund von Abweichungen zwischen vertragsärztlichen (gesicherten) Demenz-Diagnosen und der EA-Einstufung des MDK Hinweise auf die Notwendigkeit validierender Schritte für einen routinedatenbasierten Indikator zu Antipsychotikaverordnungen. Da mit den neuen Pflegegraden ab 2017 die Information über eine EA in den Abrechnungsdaten fehlt, wäre auch die alternative Verwendung des ebenso routinemäßig erstellten Datensatzes ${ }^{8}$ zur MDK-Einstufung zu prüfen. Für die Präzisierung der Kennzahl zur hausärztlichen Versorgung bietet sich etwa der Test einer M2Q-Regel (in mindestens 2 Quartalen des Referenzjahres) oder die Nutzung spezifischer EBM-Abrechnungspositionen zum Ausschluss von Notfallund Vertretungskontakten an. Ferner ließe sich auf Basis der EBM-Dokumentation zwischen fachärztlichen Heimbesuchen und Behandlungen in der Facharztpraxis unterscheiden. Zu prüfen ist außer-

\footnotetext{
8 Elektronischer Datenaustausch zwischen Kranken-/Pflegekassen (GKV/GPV) und Medizinischen Diensten der Krankenversicherung (MDK) nach den § 275 SGBV und $\S 18$ SGB XI - Technische Anlage Version 1.4 Stand: 30.11.2016, gültig ab 01.01.2017.
} 
dem, inwieweit das ASK-Konzept und das hier verwendete Indikationsset mit Blick auf multimorbide Bewohner in Pflegeheimen anzupassen und bspw. um weitere Diagnosen aus anderen ASK-Katalogen (wie durch Mangelernährung bedingte Beschwerdebilder, Dehydrierung oder Wundbrand (Purdy et al. 2009)) zu ergänzen ist. So legt Kanada z. B. eine ASK-Indikationsliste für den Qualitätsindikator zur Langzeitpflege zugrunde, der im Gegensatz zur deutschen Version Diagnosen zu Pneumonien, Grand-mal-Anfällen und Sturzverletzungen enthält (HQO 2017).

Ein weiterer wesentlicher Aspekt der methodischen Weiterentwicklung betrifft die Berücksichtigung der Risikostruktur im Pflegeheim, um faire Vergleiche von Pflegeheimen zu ermöglichen (Risikoadjustierung). Kennzahlenindividuell sind dabei folgende Fragen zu beantworten: Welche Merkmale im stationären Pflegesetting fördern das Auftreten der unerwünschten Ereignisse bzw. Auffälligkeiten? Welche dieser Merkmale sollte die Adjustierung berücksichtigen, welche sind gerade das Ziel einer Qualitätsverbesserung? Lassen sich die als relevant identifizierten Risikofaktoren für die Routinedatenanalyse übersetzen? Wie unterscheiden sich die Kennzahlen zur Qualitätsmessung, wie stark lassen sie sich durch Pflege im engen Sinne beeinflussen, wo wären sie auf einem Kontinuum zwischen Performanz- und Strukturparameter im Pflegeheim zu verorten? So handelt es sich bei einer hohen Rate an Dekubitus- und HWI-Fällen um unerwünschte gesundheitsbezogene Ereignisse für den Pflegeheimbewohner, die nachweislich durch adäquate pflegerische Leistung im engeren Sinne beeinflussbar, d. h. der Pflege zuschreibbar oder pflegesensitiv sind. Eine AntipsychotikaÜberversorgung und eine hohe Rate von ASK als vermeidbare Hospitalisierungen ergeben sich als unerwünschte Ereignisse demgegenüber aus der Schnittstellenproblematik von ärztlich-pflegerischer Performanz vor dem Hintergrund und in Wechselwirkung mit den strukturellen Rahmenbedingungen und Organisationsprozessen des Pflegeheims. Die Kennzahlen zur Anzahl der hausärztlichen Akteure sowie der Bewohner-Facharzt-Kontakte sind im Gegensatz dazu eher als Strukturparameter zu verstehen. Dies hat Auswirkungen auf die Bedeutung der Risikoadjustierung: Diese ist immer vor dem Hintergrund $\mathrm{zu}$ betrachten, welche $\mathrm{Zu}$ schreibbarkeit mit der jeweiligen Kennzahl intendiert ist. Um einen fairen Vergleich zu gewährleisten, sind beispielsweise bei pflegenahen Kennzahlen möglichst alle Faktoren zu adjustieren, die von Pflegekräften und Pflegeheim nicht zu verantworten sind. Mit Blick auf das Verfahren für die Risikoadjustierung plädieren zwei im Nachgang des Wingenfeld-Reports (2011) entstandene Expertisen mit Nachdruck für multivariate Verfahren der Adjustierung (Regressionen, hierarchisch-lineare Modelle (Stemmer und Arnold 2014; UBC 2017)). So gelten im Hinblick auf die Dekubitusentstehung neben der Mobilitätseinschränkung der Bewohner (einziges und dichotom erfasstes Adjustierungsmerkmal für Dekubitus bei Wingenfeld (2011)) zahlreiche weitere signifikante Faktoren wie Alter, Bereitschaft des Bewohners zur Dekubitusprophylaxe, terminale und andere Erkrankungen wie HWI, Wundgeschwüre, Diabetes mellitus und Parkinson sowie die Dauer von Krankenhausaufenthalten als relevante Risikofaktoren (Stemmer und Arnold 2014; UBC 2017).

Im Hinblick auf den einrichtungsübergreifenden Vergleich der HWI-Fälle als Qualitätsparameter ist ein Blasenverweilkatheter (Anteil im Pflegeheim zwischen $8,1 \%$ und $15,9 \%^{9}$ ) das wohl wichtigste, wenn auch hier nicht das einzige Adjustierungsmerkmal: Im Krankenhaus gelten bis zu $80 \%$ aller Harnwegsinfektionen als katheter-assoziiert (CAUTI) (Loveday et al. 2014). Die Harnwegsinfektion ist in diesem Sinne eine Komplikation der Katheterisierung (KRINKO 2015); $70 \%$ aller CAUTIS unabhängig vom Versorgungssetting, in dem sie auftreten - sind KRINKO zufolge vermeidbar, primär durch Indikationsassessment, Vermeidung nicht indizierter Katheterisierungen, unnötig lange Verweildauern, adäquate Hygienemaßnahmen beim Legen und bei der Pflege des Kathetersystems sowie die Gewährleistung eines geschlossenen Drainagesystems (Engelhart et al. 2009; KRINKO 2015; Wischnewski et al. 2011; ZQP 2012). Für die Kennzahl zur Antipsychotika-Verordnung im Pflegeheim sind primär die unterschiedlichen einrichtungsspezifischen Prävalenzraten von psychischen und

9 10,2 \% gemäß Wischnewski (2011), 8,1 \% gemäß Engelhart (2009), 15,9\% gemäß ZQP (2012). 
Verhaltenssymptomen (Indikationen für Antipsychotika) zu adjustieren. Da psychische und Verhaltenssymptome der Demenz in der ICD-Klassifikation - trotz ihrer Häufigkeit - nur indirekt Berücksichtigung finden (Zaudig 2000), wäre die Nutzung von Informationen des MDK-Pflegeberichts (Modul s3: Verhaltensweisen und psychische Problemlagen) zu prüfen.

Für beide Kennzahlen zur ärztlichen Versorgung wäre zu diskutieren, inwiefern hier von einer "Zuschreibbarkeit« ausgegangen werden kann oder ob es sich um reine Strukturparameter handelt. Stellt man sich auf den Standpunkt, dass die Pflegeheimleitung keinen Einfluss auf die Zahl der im Pflegeheim tätigen Ärzte hat, wäre eine Adjustierung nur interessant, um regionale Unterschiede besser zu verstehen Stellt man sich auf den Standpunkt, dass sich ein qualitativ gut geführtes Pflegeheim gerade dadurch auszeichnet, dass Pflegeheime aktiv mit Ärzten der Fachgruppe Neurologen, Psychiater und Nervenärzte kooperieren, müsste neben erkrankungsbedingten Einflussgrößen sicher u. a. auch die regionale Versorgungsdichte als Adjustierungsmerkmal herangezogen werden.

Bei aller Bedeutung einer angemessenen Risikoadjustierung im Rahmen der einrichtungsvergleichenden Qualitätsmessung auf Basis von Routinedaten bleibt festzuhalten, dass die Entwicklung eines methodisch in der Regel hochkomplexen und anspruchsvollen statistischen Adjustierungsmodells praktikabel bleibt. Die - methodisch inhärenten - Grenzen der Risikoadjustierung dürfen der hier anvisierten Qualitätsmessung jedoch nicht per se im Wege stehen. Erstens zeigen Erfahrungen, dass sowohl die Varianz als auch die grundlegende Bewertung der Einrichtungen auch nach Adjustierung im Großen und Ganzen bestehen bleiben. Zweitens ist die hier angestrebte Nutzung der Kennzahlen primär für die interne Qualitätstransparenz und nicht für die externe Qualitätsberichterstattung gedacht.

Neben einer Adjustierung der Ergebnisse sollte insbesondere der Echtbetrieb der routinedatenbasierten Qualitätsmessung im stationären Pflegesetting das sogenannte Fallzahl-Prävalenz-Problem im Blick haben: Eine geringe Häufigkeit von unerwünschten Ereignissen in Pflegeeinrichtungen mit wenigen Bewohnern beeinträchtigt die Reliabilität der Qualitätsmessung. Zur Begrenzung der Problematik schlossen die vorliegenden Analysen in einem ersten Schritt Pflegeheime mit weniger als 20 AOK-Versicherten je Quartal aus der Stichprobe aus. Die Entwicklung von geeigneten Indizes ist eine Möglichkeit, die Prävalenz der betrachteten Ereignisse, die Fallzahl und damit die Zuverlässigkeit der Messung zu erhöhen.

\subsection{Fazit}

Die Messung von Qualität in der stationären Langzeitpflege auf Basis von Routinedaten - das zeigt der Beitrag - ist machbar und weist auf erhebliche Unterschiede zwischen den Pflegeheimen hin. Die hier gewonnenen kennzahlenspezifischen Ergebnisse unterstreichen die Relevanz der ausgewählten Versorgungsaspekte und den Bedarf an versorgungsoptimierenden Maßnahmen. „Qualität« ist hier bewusst aus der Perspektive des Pflegeheimbewohners operationalisiert, d. h. seine Versorgungsqualität wurde bewertet. Welchen Akteuren die Ergebnisse zuschreibbar sind - ob nun primär den einzelnen Pflegekräften bzw. der Pflegeheimleitung oder den Ärzten -, wurde hier zunächst nicht in den Fokus gerückt. Gerade weil die Versorgungsdefizite multikausal und berufsgruppenübergreifend $\mathrm{zu}$ verorten sind, sollte sich auch die Herangehensweise bei der Messung der Versorgungsqualität daran orientieren. Gleichwohl muss bei der Weiterentwicklung der Indikatoren die Frage der jeweiligen Risikoadjustierung vor dem Hintergrund des intendierten Aussagegehalts bzw. intendierter Zuschreibungen in den Blick genommen werden. In der Gesamtschau stimmen die Ergebnisse - trotz des benannten methodischen Weiterentwicklungsbedarfs - optimistisch für eine weitere Schärfung der hier verwendeten routinedatenbasierter Qualitätsindikatoren bzw. für die Entwicklung weiterer Indikatoren. Das Potenzial derartiger sozialleistungsträger- und professionsübergreifenden Qualitätsindikatoren für die stationäre Langzeitpflege ist erheblich: Die routinedatenbasierte Umsetzung ist mit vergleichsweise geringem Aufwand verbunden; eine weitere Dokumentation durch die Pflegeheime ist nicht notwendig. Die Indikatoren schaffen Awareness für konkrete Pflegedefizite und können 
die pflegeheiminterne Qualitätssicherung, Qualitätszirkel mit weiteren Berufsgruppen sowie auch Vertragsverhandlungen zwischen Pflegekassen und Einrichtungen empirisch fundieren. Angestrebt ist nicht das Festlegen (normativ begründeter) referentieller Geltungsbereiche für »schlechte« Performanz, sondern der relationale Vergleich über die Pflegeheime hinweg und langfristig auch über die Zeit. Eine ggf. mögliche weitere Nutzung einiger der Indikatoren für die externe Qualitätsberichterstattung ist erst zu einem späteren Zeitpunkt - bei hinreichender Reifung der methodischen Fundierung der Indikatoren - zu diskutieren. Was im Krankenhausbereich mit dem im WIdO entwickelten QSR-Verfahren ${ }^{10}$ bereits seit Jahren etabliert ist, erweist sich im Pflegebereich national und international als Neuland. Auf der Agenda für eine zukünftige routinedatenbasierte Qualitätsmessung in der Pflege ist mit den hier vorgelegten Analysen jedoch der erste Schritt getan.

10 http://www.qualitaetssicherung-mit-routinedaten.de/

\subsection{Anhang}

- Tab. 10.9 Verbandsmaterial für die Wundversorgung bei Dekubitus - einbezogene Produktgruppen nach PZN-Klassifikation zur Identifizierung der Dekubitusfälle auf Routinedatenbasis

\begin{tabular}{|c|c|}
\hline PZN-Klassifikation: Gruppe & $\begin{array}{l}\text { Anteil an allen einbezogenen PZN } \\
\text { (in } \% \text {, gesamt }=4.913 \text { ) }\end{array}$ \\
\hline Schaumverbände feinporig & 37,2 \\
\hline Superabsorber/Vlieskompresse mit Superabsorber & 10,1 \\
\hline Hydrokolloide normal & 9,9 \\
\hline Alginate & 8,3 \\
\hline Folien/ semipermeable Transparentfolien & 7,4 \\
\hline Hydrogel & 6,2 \\
\hline Hydrofaser/-fiber/ Aquafaser & 4,6 \\
\hline Aktive Wundauflagen & 4,6 \\
\hline Aktivkohleverbände & 2,1 \\
\hline Hydrokolloidähnliche Wundauflagen & 1,5 \\
\hline Wundspülungen konserviert & 1,5 \\
\hline Honig-Produkte & 1,5 \\
\hline Hydrophobe Wundauflagen & 1,4 \\
\hline Saugspülkörper zur Nasstherapie & 1,2 \\
\hline Sonstige Wundauflagen & 0,7 \\
\hline Produkte zur Unterstützung der Wundreinigung & 0,6 \\
\hline Moderne Post-OP-Verbände & 0,5 \\
\hline Wundauflagen aus feuchter Zellulose/ Sonstige Wundauflagen & 0,4 \\
\hline Hautschutzpräparate & 0,2 \\
\hline Produkte für Spalthautentnahmestellen & 0,1 \\
\hline Quelle: WidO & \\
\hline
\end{tabular}


- Tab. 10.10 Indizierte Antibiotika zur Therapie der Harnwegsinfektion - einbezogene Wirkstoffe nach ATC-Klassifikation zur Identifizierung der HWI auf Routinedatenbasis

\begin{tabular}{|c|c|c|c|}
\hline ATC-Code & Wirkstoff & ATC-Code & Wirkstoff \\
\hline J01CG01 & Sulbactam & J01DD13 & Cefpodoxim \\
\hline J01CR04 & Sultamicillin & J01DD14 & Ceftibuten \\
\hline J01CR21 & Ampicillin und Sulbactam & J01DE01 & Cefepim \\
\hline J01CR22 & Amoxicillin und Clavulansäure & J01DH02 & Meropenem \\
\hline J01EA01 & Trimethoprim & J01DH03 & Ertapenem \\
\hline J01EE01 & Sulfamethoxazol und Trimethoprim & J01DH21 & Imipenem und Cilastatin \\
\hline J01XE01 & Nitrofurantoin & J01EE01 & Sulfamethoxazol und Trimethoprim \\
\hline J01XE51 & Nitrofurantoin, Kombinationen & J01GB01 & Tobramycin \\
\hline J01XX01 & Fosfomycin & J01GB03 & Gentamicin \\
\hline J01XX07 & Nitroxolin & J01GB06 & Amikacin \\
\hline J01CA01 & Ampicillin & J01MA01 & Ofloxacin \\
\hline J01CA04 & Amoxicillin & J01MA02 & Ciprofloxacin \\
\hline J01CR25 & Piperacillin und Tazobactam & J01MA04 & Enoxacin \\
\hline J01DC02 & Cefuroxim & J01MA06 & Norfloxacin \\
\hline J01DC04 & Cefaclor & J01MA12 & Levofloxacin \\
\hline J01DD01 & Cefotaxim & J01XX01 & Fosfomycin \\
\hline J01DD02 & Ceftazidim & J01MA14 & Moxifloxacin \\
\hline J01DD04 & Ceftriaxon & J01CA10 & Mezlocillin \\
\hline J01DD08 & Cefixim & J01CA12 & Piperacillin \\
\hline Quelle: WIdC & & & \\
\hline
\end{tabular}

- Tab. 10.11 Haus- und fachärztliche Versorgung im Pflegeheim - einbezogene Facharztgruppen zur Identifizierung der Hausärzte und der Neurologen, Nervenärzte und Psychiater auf Routinedatenbasis

\begin{tabular}{|l|l|l|l|l|l|l|l|}
\hline Fachgruppe & Bezeichnung \\
\hline 01 & Hausarzt: Facharzt Allgemeinmedizin/ Innere und Allgemeinmedizin, Schwerpunkt Geriatrie \\
\hline 02 & Hausarzt/Praktischer Arzt, obsolet \\
\hline 03 & Hausarzt: Facharzt Innere Medizin und Schwerpunkt gesamte Innere Medizin \\
\hline Neurologen, Nervenärzte, Psychiater \\
\hline 51 & $\begin{array}{l}\text { Facharzt Nervenheilkunde, obsolet; Facharzt Neurologie und Psychiatrie, obsolet; } \\
\text { Facharzt Neurologie, Psychiatrie und Psychotherapie, kammerindividuell, obsolet }\end{array}$ \\
\hline 53 & Facharzt Neurologie, Schwerpunkt Geriatrie \\
\hline 58 & Facharzt Psychiatrie und Psychotherapie; Facharzt Psychiatrie, obsolet; Schwerpunkt Geriatrie \\
\hline 59 & Schwerpunkt Forensische Psychiatrie \\
\hline Quelle: WIdO, in Anlehnung an KBV 2017 \\
\hline
\end{tabular}


Open Access Dieses Kapitel wird unter der Creative Commons Namensnennung 4.0 International Lizenz (http://creativecommons. org/licenses/by/4.0/deed.de) veröffentlicht, welche die Nutzung, Vervielfältigung, Bearbeitung, Verbreitung und Wiedergabe in jeglichem Medium und Format erlaubt, sofern Sie den/die ursprünglichen Autor(en) und die Quelle ordnungsgemäß nennen, einen Link zur Creative Commons Lizenz beifügen und angeben, ob Änderungen vorgenommen wurden.

Die in diesem Kapitel enthaltenen Bilder und sonstiges Drittmaterial unterliegen ebenfalls der genannten Creative Commons Lizenz, sofern sich aus der Abbildungslegende nichts anderes ergibt. Sofern das betreffende Material nicht unter der genannten Creative Commons Lizenz steht und die betreffende Handlung nicht nach gesetzlichen Vorschriften erlaubt ist, ist für die oben aufgeführten Weiterverwendungen des Materials die Einwilligung des jeweiligen Rechteinhabers einzuholen.

\section{Literatur}

Albrecht M, Nolting H-D, Schliwen A (2012) Konzept zur Neuordnung der ärztlichen Bedarfsplanung. G\&S Gesundheits- und Sozialpolitik 66:29-35. doi:10.5771/1611-58212012-5-29

Balzer K, Butz S, Bentzel J, Boulkhemair D, Lühmann D (2013) Beschreibung und Bewertung der fachärztlichen Versorgung von Pflegeheimbewohnern in Deutschland. Schriftenreihe Health Technology Assessment 125:1-320. doi:10.3205/hta000108L

Bartholomeyczik S et al. (2006) Rahmenempfehlungen zum Umgang mit herausforderndem Verhalten bei Menschen mit Demenz in der stationären Altenhilfe. http://siegel. dggpp.de/Rahmenempfehlungen_herausf_Verhaltene. pdf. Zugegriffen: 07. Juli 2017

Bartholomeyczik S et al. (2010) Sachbericht zum Projekt »Interdisziplinäre Implementierung von Qualitätsinstrumenten zur Versorgung von Menschen mit Demenz in Altenheimen« (InDemA). https://www.uni-wh.de/fileadmin/ media/u/forschung/izvf/InDemA_Abschlussbericht_incl. Anhang_07.10.10.pdf. Zugegriffen: 25. Oktober 2016

Becher KF, Klempien I, Wiedemann A (2015) Harnwegsinfekte im Alter. Zeitschrift für Gerontologie und Geriatrie 48:588-594

Bergner S (2016) Psychopharmaka Verschreibungsmuster und unerwünschte Wirkung bei Älteren. Dissertationsarbeit in Vorbereitung. Universität Bonn. www.amts-ampel.de. Zugegriffen: 20. November 2016

Burgdorf F, Sundmacher L (2014) Potenziell vermeidbare Krankenhausfälle in Deutschland Analyse von Einflussfaktoren auf die Raten ambulant-sensitiver Krankenhauseinweisungen. Deutsches Ärzteblatt International 13:215-223

Burke RE, Rooks SP, Levy C, Schwartz R, Ginde AA (2015) Identifying potentially preventable emergency department visits by nursing home residents in the United States. J Am Med Dir Assoc 5:395-399

Cerejeira J, Lagarto L, Mukaetova-Ladinska EB (2012) Behavioral and psychological symptoms of dementia. Frontiers in Neurology 3. doi:doi.org/10.3389/fneur.2012.00073

CMS (2016) Long-Term Care Facility Resident Assessment Instrument 3.0 User's Manual - Version 1.14 October 2016. https://downloads.cms.gov/mwg-internal/de5fs-
23hu73g7/progress?id=xSI2pmZbPDTK3A4QcySjawZiczU w1VaitkegsC5GJ7A,\&dl. Zugegriffen: 20. Januar 2017

Coon JT et al. (2014) Interventions to Reduce Inappropriate Prescribing of Antipsychotic Medications in People With Dementia Resident in Care Homes: A Systematic Review. JAMDA 15:706-718 doi:10.1016/j.jamda.2014.06.012

Declercq T, Petrovic M, Azermai M, Vander Stichele R, De Sutter Al, van Driel ML, Christiaens T (2013) Withdrawal versus continuation of chronic antipsychotic drugs for behavioural and psychological symptoms in older people with dementia. Cochrane Database Syst Rev:Cd007726. doi:10.1002/14651858.CD007726.pub2

DGPPN und DGN (2016) S3-Leitlinie »Demenzen« - Langversion - Januar 2016. https://www.dgppn.de/_Resources/Persistent/ade50e44afc7eb8024e7f65ed3f44e995583c3a0/ S3-LL-Demenzen-240116.pdf. Zugegriffen: 22. Januar 2018

DGU (2017) Interdisziplinäre S3 Leitlinie - Epidemiologie, Diagnostik, Therapie, Prävention und Management unkomplizierter, bakterieller, ambulant erworbener Harnwegsinfektionen bei erwachsenen Patienten - Aktualisierung 2017. http://www.awmf.org/uploads/tx_ szleitlinien/043-044k_S3_Harnwegsinfektionen_2017-05. pdf. Zugegriffen: 02. März 2018

Dickson K, Lafortune L, Kavanagh J, Thomas J, Mays N, Erens B (2012) Non-drug treatments for symptoms in dementia: an overview of systematic reviews of non-pharmacological interventions in the management of neuropsychiatric symptoms and challenging behaviours in patients with dementia. Policy Research Unit in Policy Innovation Research, London

DKG, GKV-Spitzenverband, PKV, InEK GmbH (2017) Deutsche Kodierrichtlinien. Allgemeine und Spezielle Kodierrichtlinien für die Verschlüsselung von Krankheiten und Prozeduren. www.dkgev.de. Zugegriffen: 05. März 2018

DNQP (2015) Expertenstandard Pflege von Menschen mit chronischen Wunden - 1. Aktualisierung. Schriftenreihe des Deutschen Netzwerks für Qualitätsentwicklung in der Pflege, Osnabrück

DNQP (2017) Expertenstandard Dekubitusprophylaxe in der Pflege - 2. Aktualisierung 2017. Osnabrück

Engelhart S, Lauer A, Simon D, Exner U, Heudorf U, Exner M (2009) Wiederholte Prävalenzuntersuchungen Pflegeheimassoziierter Infektionen als Instrument zur Erfassung der 
hygienischen Ergebnisqualität. Bundesgesundheitsblatt

- Gesundheitsforschung - Gesundheitsschutz 52:936-944

Fischbach D (2016) Krankenhauskosten ambulant-sensitiver Krankenhausfälle in Deutschland. Gesundheitswesen

3:168-174

Fünfstück R (2011) Harnwegsinfektionen bei alten Menschen. http://www.p-e-g.de/archiv_tmp/bad_honnef_symposium_2011/Fuenfstueck.pdf. Zugegriffen: 02. März 2018

Gallagher RM, Rowell PA (2003) Claiming the future of nursing through nursing-sensitive quality indicators. Nurs Adm Q 4:273-284

Gutzmann H, Schäufele M, Kessler E-M, Rapp MA (2017) Psychiatrische und psychotherapeutische Versorgung von Pflegebedürftigen. In: Jacobs K, Kuhlmey A, Greß S, Klauber J, Schwinger A (Hrsg) Pflege-Report 2017 Schwerpunkt: Die Versorgung der Pflegebedürftigen. Schattauer Verlag, Stuttgart, S 107-117

Hasseler M, Stemmer R, Macsenaere M, Arnold J, WeidekampMaicher M (2016) Entwicklung eines wissenschaftlich basierten Qualitätsverständnisses für die Pflege- und Lebensqualität. Abschlussbericht. https://www.gkv-spitzenverband.de/mwg-internal/de5fs23hu73g7/ progress?id=Vu9ZwuSY4iHzGk-RNvy83n_ jWqCBWUolaOVCyk4Yrf8,\&dl. Zugegriffen: 02. Januar 2018

Heslop L (2014) Nursing-sensitive indicators: a concept analysis. J Adv Nurs 11:2469-2482

HQO (2015) LTC Indicator Review Report. The review and selection of indicators for long-term care public reporting. November 2015. http://www.hqontario.ca/SystemPerformance/Measuring-System-Performance/Measuring-Long-Term-Care-Homes. Zugegriffen: 06. März 2018 HQO (2016) Long-Term Care Impressions and Observations 2015/16 Quality Improvement Plans. www.hqontario.ca

IGES (2012) Bewertung der Kodierqualität von vertragsärztlichen Diagnosen - Eine Studie im Auftrag des GKVSpitzenverbands in Kooperation mit der BARMER GEK. https://www.gkv-spitzenverband.de/media/dokumente/ krankenversicherung_1/aerztliche_versorgung/verguetung_und_leistungen/klassifikationsverfahren/9_Endbericht_Kodierqualitaet_Hauptstudie_2012_12-19.pdf. Zugegriffen: 02. März 2018

Inspectie voor de Gezondheidszorg (2013) Kwaliteitsdocument 2013. Verpleging, Verzorging en Zorg Thuis. http:// www.igz.nl/Images/Kwaliteitsdocument-VVT-2013_ tcm294-329148.pdf. Zugegriffen: 07. Oktober 2016

James AJ (2011) Herausforderndes Verhalten bei Menschen mit Demenz - Einschätzen, verstehen und behhandeln, 1. Auflage. Verlag Hans Huber, Bern

Kirkham J, Velkers C, Maxwell C, Gill S, Rochon P, Seitz D (2017) Antipsychotic Use in Dementia: Is There a Problem and Are There Solutions? The Canadian Journal of Psychiatry 62:170-181. doi:10.1177/0706743716673321

Kirsebom M, Hedström M, Pöder U, Wadensten B (2015) Transfer of nursing home residents to emergency departments: organizational differences between nursing homes with high vs. low transfer rates. Nursing Open 4:41-48. doi:10.1002/nop2.68
Kleina T, Horn A, Suhr R, Schaeffer D (2017) Zur Entwicklung der ärztlichen Versorgung in stationären Pflegeeinrichtungen - Ergebnisse einer empirischen Untersuchung. [Current Status of Medical Care for Nursing Home Residents in Germany - Results of an Empirical Study] 79:382-387. doi:10.1055/s-0035-1549971

Klose J, Rehbein I (2016) Ärzteatlas 2016 - Daten zur Versorgungsdichte von Vertragsärzten. https://www.wido.de/ fileadmin/wido/downloads/pdf_ambulaten_versorg/ wido_amb_pub-aerzteatlas2016_0716.pdf. Zugegriffen: 02. März 2018

KRINKO (2001) Mitteilung der Kommission für Krankenhaushygiene und Infektionsprävention zur Surveillance (Erfassung und Bewertung) von nosokomialen Infektionen (Umsetzung von § 23 IfSG). Bundesgesundheitsblatt Gesundheitsforschung - Gesundheitsschutz 44:523-536

KRINKO (2015) Prävention und Kontrolle Katheter-assoziierter Harnwegsinfektionen. Empfehlung der Kommission für Krankenhaushygiene und Infektionsprävention (KRINKO) beim Robert Koch-Institut. Bundesgesundheitsblatt Gesundheitsforschung - Gesundheitsschutz 58:641-650

KRINKO (2016) Händehygiene in Einrichtungen des Gesundheitswesens Empfehlung der Kommission für Krankenhaushygiene und Infektionsprävention (KRINKO) beim Robert Koch-Institut (RKI). Bundesgesundheitsblatt Gesundheitsforschung - Gesundheitsschutz: 59:11891220

Kuhlmey A, Sibbel R, Fischer T (2010) Wirksamkeit der deutschen Version der Serial Trial Intervention zur ursachebezogenen Reduktion von herausforderndem Verhalten bei Menschen mit Demenz (STI - D) gefördert durch das Bundesministerium für Gesundheit im Rahmen des «Leuchtturmprojekt Demenz«. https://medsoz.charite. de/fileadmin/user_upload/microsites/m_cc01/medsoz/ STI-D_Projektbericht.pdf. Zugegriffen: 10. Juli 2017

Leffmann C, Anders J, Heinemann A, Leutenegger M, Pröfener F (2005) Dekubitus. Geundheitsberichterstattung des Bundes. Oktoberdruck, Berlin

Loveday HP et al. (2014) Epic3: national evidencebased guidelines for preventing healthcare-associated infections in NHS hospitals in England. J Hosp Infect 1-70

Mauleon dA et al. (2014) Associated Factors with antipsychotic use in Long-Term Institutional Care in eight European Countries: results from the RightTimePlaceCare Study. JAMDA 15:812-818. doi:10.1016/j.jamda.201406.015

MDS (2018) Qualität in der ambulanten und stationären Pflege. 5. Pflege-Qualitätsbericht des MDS nach §114a Abs. 6 SGB XI https://www.mds-ev.de/richtlinien-publikationen/pflegeversicherung/mds-pflege-qualitaetsberichte.html. Zugegriffen: 05. März 2018

Molter-Bock E, Hasford J, Pfundstein T (2006) Psychopharmakologische Behandlungspraxis in Münchener Altenpflegeheimen. Z Gerontol Geriat 39:336-343

Nakrem S, Vinsnes AG, Harkless GE, Paulsen B, Seim A (2009) Nursing sensitive quality indicators for nursing home care: International review of literature, policy and practice. Int J Nurs Stud 6:848-857 
Naumann C, Augustin U, Sundmacher L (2015) Ambulantsensitive Krankenhausfälle in Deutschland: Eine Analyse auf Kreisebene für die Jahre 2006-2009.

Gesundheitswesen:e91-e105

Nitschke I, Micheelis W (2016) Krankheits- und Versorgungsprävalenzen bei Älteren Senioren mit Pflegebedarf. In: Jordan R, Micheelis W, Institut der Deutschen Zahnärzte (Hrsg) Fünfte Deutsche Mundgesundheitsstudie (DMS V). Deutscher Zahnärzte Verlag, Köln, S 557-578

NPUAP, EPUAP, PPPIA (2014) Prevention and Treatment of Pressure Ulcers. Quick Reference Guide. 2. Auflage. www.epuap.org. Zugegriffen: 05. März 2018

Purdy S, Griffin T, Salisbury C, Sharp D (2009) Ambulatory care sensitive conditions: terminology and disease coding need to be more specific to aid policy makers and clinicians. Public health 123:169-173. doi:10.1016/j.puhe. 2008.11.001

Ramroth H, Specht-Leible N, König HH, Brenner H (2006) Hospitalizations during the last months of life of nursing home residents: a retrospective cohort study from Germany. BMC Health Serv Res 6:https://doi. org/10.1186/1472-6963-1186-1170

Richter T, Mann E, Meyer G, Haastert B, Kopke S (2012) Prevalence of psychotropic medication use among German and Austrian nursing home residents: a comparison of 3 cohorts. Journal of the American Medical Directors Association 13:187.e187-187.e113. doi:10.1016/j.jamda. 2011.03.007

Rothgang H, Borchert L, Müller R, Unger R (2008) GEK-Pflegereport 2008. Schwerpunkt: Medizinische Versorgung in Pflegeheimen. Asgard-Verlag, St. Augustin

Rothgang H, Müller R, Mundhenk R, Unger R (2014) BARMER GEK Pflegereport 2014: Schwerpunkt: Zahnärztliche Versorgung Pflegebedürftiger. Asgard-Verlag, St. Augustin

Ruscher C, Kraus-Haas M, Nassauer A, Mielke M (2015) Healthcare-associated infections and antimicrobial use in long term care facilities (HALT-2). Deutsche Ergebnisse der zweiten europäischen Prävalenzerhebung. Bundesgesundheitsblatt - Gesundheitsforschung - Gesundheitsschutz 58:436-451

Ruscher C, Schaumann R, Mielke M (2012) Herausforderungen durch Infektionen und mehrfachresistente Bakterien bei alten Menschen in Heimen. Bundesgesundheitsblatt - Gesundheitsforschung - Gesundheitsschutz 55:14441452. doi:0.1007/s00103-012-1555-7

Savitz LA, Jones CB, Bernard S (2005) Quality Indicators Sensitive to Nurse Staffing in Acute Care Settings. In: Henriksen K, Battles JB, Marks ES, Lewin DI (Hrsg) Advances in Patient Safety: From Research to Implementation. Agency for Healthcare Research and Quality Rockville, S 375-385

Schulz A, Doblhammer G (2012) Aktueller und zukünftiger Krankenbestand von Demenz in Deutschland auf Basis der Routinedaten der AOK. In: Günster C, Klose J, Schmacke N (Hrsg) Versorgungs-Report 2012. Schwerpunkt: Gesundheit im Alter. Schattauer, Stuttgart, S 161-171
Schwarzkopf L, Holle R, Schunk M (2017) Effects of Nursing Home Residency on Diabetes Care in Individuals with Dementia: An Explorative Analysis Based on German Claims Data. Dement Geriatr Cogn Dis Extra 7:41-51

Schwinger A, Jürchott K, Tsiasioti C (2017a) Pflegebedürftigkeit in Deutschland. In: Jacobs K, Kuhlmey A, Greß S, Klauber J, Schwinger A (Hrsg) Pflege-Report 2017 Schwerpunkt: Die Versorgung der Pflegebedürftigen. Schattauer, Stuttgart, S 255-303

Schwinger A, Jürchott K, Tsiasioti C, Rehbein I (2016) Pflegebedürftigkeit in Deutschland. In: Jacobs K, Kuhlmey A, Greß S, Klauber J, Schwinger A (Hrsg) Pflege-Report 2016 - Schwerpunkt: Die Pflegenden im Fokus. Schattauer, Stuttgart, S 275-328

Schwinger A, Tsiasioti C, Klauber J (2017b) Herausforderndes Verhalten bei Demenz: Die Sicht der Pflege. In: Jacobs K, Kuhlmey A, Greß S, Klauber J, Schwinger A (Hrsg) PflegeReport 2017 - Schwerpunkt: Die Versorgung der Pflegebedürftigen. Schattauer, Stuttgart, S 131-151

Statistisches Bundesamt (2017) Pflegestatistik 2015 - Pflege im Rahmen der Pflegeversicherung - Deutschlandergebnisse. https://www.destatis.de/DE/Publikationen/ Thematisch/Gesundheit/Pflege/PflegeDeutschlandergebnisse5224001159004.pdf?_blob=publicationFile. Zugegriffen: 02. März 2018

Stemmer R, Arnold J (2014) Expertise zur «Eignung von Indikatoren zur Messung und Darstellung von Ergebnisqualität in der stationären Pflege im Bereich der sozialen Pflegeversicherung". https://www.ikj-mainz.de/tl_files/ Downloads/Pulikationen/Expertise \%20Ergebnisqualitaetsindikatoren \%20stationaere \%20Pflege_Stemmer \%20 \&\%20Arnold_2014.pdf. Zugegriffen: 02. Januar 2018

Sundmacher L, Schüttig W (2015) Which hospitalisations are ambulatory care-sensitive, to what degree, and how could the rates be reduced? Results of a group consensus study in Germany. Health Policy 11:1415-1423

Sveriges Kommuner och Landsting och Socialstyrelsen (2015) Beskrivning av indikatorerna i Öppna jämförelser - Vård och omsorg om äldre 2015. http://www.socialstyrelsen. se/SiteCollectionDocuments/2016-3-1-Bilaga \%20 2-Beskrivning \%20av \%20indikatorerna.pdf. Zugegriffen: 17. Januar 2017

Temple BA, Krishnan P, O'Connell B, Grant LG, Demczuk' L (2017) Emergency department interventions for persons with dementia presenting with ambulatory care-sensitive conditions: a scoping review protocol. JBI Database System Rev Implement Rep 2:196-201

Thürmann P (2017) Einsatz von Psychopharmaka bei Pflegebedürftigen. In: Jacobs K, Kuhlmey A, Greß S, Klauber J, Schwinger A (Hrsg) Pflege-Report 2017 - Schwerpunkt: Die Versorgung der Pflegebedürftigen. Schattauer, Stuttgart, S 119-129

Thürmann P, Jaehde U, Bernard S, Schröder F (2010) Abschlussbericht zum Projekt Arzneimitteltherapiesicherheit in Alten- und Pflegeheimen: Querschnittsanalyse und Machbarkeit eines multidisziplinären Ansatzes. https://www.bundesgesundheitsministerium.de/filead- 
min/dateien/Publikationen/Gesundheit/Sonstiges/

Abschlussbericht_Arzneimitteltherapiesicherheit_in_Al-

ten-_und_Pflegeheimen_Querschnittsanalyse_und_

Machbarkeit_eines_multidisziplinaeren_Ansatzes.pdf.

Zugegriffen: 25. Oktober 2016

UBC (2017) Abschlussbericht zum Forschungsprojekt Modellhafte Pilotierung von Indikatoren in der stationären Pflege (MoPIP). https://www.gkv-spitzenverband.de/ mwg-internal/de5fs23hu73g7/progress?id=TK42E98TvcE dV7cFjeP9Ef9UBrm5M9E-WkaQRra760Q,\&dl. Zugegriffen: 02. Januar 2018

Wiese C, Ittner K, Lassen C (2016) Weniger ist oft mehr. Palliativer Notfall bei geriatrischen Patienten. MMW Fortschritte der Medizin 158:82-86

Wingenfeld K, Kleina T, Franz S, Engels D, Mehlan S, Engel H (2011) Entwicklung und Erprobung von Instrumenten zur Beurteilung der Ergebnisqualität in der stationären Altenhilfe. Abschlussbericht. https://www.bmfsfj.de/blob /93206/2dda7f65c418478da3260d2f7996daa2/abschlussbericht-stationaere-altenhilfe-data.pdf. Zugegriffen: 02. Februar 2018

Wischnewski N, Mielke M, Wendt C (2011) Healthcare-associated infections in long-term care facilities (HALT) - Ergebnisse aus Deutschland im Rahmen einer europäischen Prävalenzstudie. Bundesgesundheitsblatt - Gesundheitsforschung - Gesundheitsschutz 54:1147-1152. doi:10.1007/s00103-011-1363-5

ZQP (2012) Qualität und Gesundheit in der stationären Altenhilfe - eine empirische Bestandsaufnahme. Abschlussbericht. https://www.zqp.de/wp-content/uploads/ Abschlussbericht_Qualitaet_Gesundheit_Stationaeren_ Altenhilfe.pdf 